\title{
Einstein coefficients and equilibrium formalism for tachyon radiation
}

\author{
Roman Tomaschitz* \\ Department of Physics, Hiroshima University, 1-3-1 Kagami-yama, Higashi-Hiroshima 739-8526, Japan
}

Received 23 June 2000; received in revised form 22 September 2000

\begin{abstract}
The spectral energy density of an ideal Bose gas of superluminal particles (tachyons) is derived. To this end, we consider atoms in equilibrium with tachyon radiation, study spontaneous and induced transitions effected by tachyons, calculate the Einstein coefficients, all semiclassically, and obtain, by detailed balancing, the equilibrium distribution of the tachyon gas. Tachyons are described by a real Proca field with negative mass square, coupled to a current of subluminal matter. Atomic transitions induced by tachyons are compared to photonic ones, and the tachyonic analog to the photoelectric effect is discussed. The cosmic tachyon background is scrutinized in detail; high- and low-temperature expansions of the internal energy, the entropy, the heat capacities, and the number density are compared with the corresponding quantities of the photon background. The negative mass square in the wave equation changes the frequency scaling in the Rayleigh-Jeans law, and there are also significant changes in the low-temperature regime, in particular in the caloric and thermal equations of state. Quantitative estimates on the tachyon background and on Rydberg transitions induced by tachyon radiation are derived.

(C) 2001 Elsevier Science B.V. All rights reserved.
\end{abstract}

PACS: 98.70.Vc; 05.30.Jp; 03.65.Sq; 32.70.Cs; 34.60.+z

Keywords: Superluminal black-body radiation; Tachyonic background radiation; Ether; Proca equation; Rydberg atoms

\section{Introduction}

Modern theories of superluminal motion are based on the formalism of classical relativistic mechanics. Faster-than-light particles (tachyons) are usually introduced as an extension of the relativistic particle concept, as particles with negative mass square,

* Fax: +81-824-240717.

E-mail address: roman@fusion.sci.hiroshima-u.ac.jp (R. Tomaschitz). 
or, if one prefers, imaginary mass. In the relativistic Hamilton-Jacobi equation, this just means to assume $m^{2}<0$ without further alterations, and if tachyons are supposed to carry electric charge, then their coupling to the electromagnetic potential is usually effected by minimal substitution. Des Coudres's and Sommerfeld's pre-relativistic studies of superluminal motion [1,2] aimed at accelerating electrons beyond the speed of light by means of electromagnetic fields, but otherwise their view of tachyons as point particles coupled in the usual way to the electromagnetic field was taken over by modern authors, cf. Refs. [3-10] and [11-22] for related discussions on causality, relativity, and synchronicity in few-body and statistical systems.

In this article, a different approach to superluminal motion is investigated. Superluminal wave propagation is modeled in analogy to classical electrodynamics, by a Proca equation with negative mass square [23-25], very contrary to the prevailing view of tachyons as electrically charged point particles with imaginary mass. The superluminal wave modes of the tachyon field are coupled to a current of subluminal massive particles. Like the electromagnetic field, the tachyon field is conformally coupled to the background geometry [26,27] so that the frequencies of the spectral elementary waves scale inversely proportional to the curvature radius of the cosmic 3-space. As for the cosmic tachyon background radiation, this conformal scaling allows, despite the time variation of the background geometry, to use the thermodynamic equilibrium formalism, and to scale the time dependence of the eigenmodes into the temperature variable, which becomes in this way a function of cosmic time. High- and low-temperature expansions for the internal energy, entropy, pressure, heat capacities, and the tachyon density are derived. In the high-temperature regime, one recovers in leading asymptotic order the familiar results for the photon background, but the low-temperature behavior of these quantities gets completely modified by the tachyon mass.

In Section 2, we sketch the wave equation for tachyons, the spectral modes, the tachyonic energy density and flux, and we discuss the coupling of subluminal matter (point particles and wave functions) to the tachyon field. In Section 3, we study tachyonic radiation processes, spontaneous and induced emission and induced absorption. We calculate the Einstein coefficients, give in this context a semiclassical derivation of the tachyonic equilibrium distribution, and discuss the tachyonic analog to the photoelectric effect.

In Section 4, we calculate the spectral energy density of a free tachyon gas and discuss in some detail the high- and low-temperature expansions of the internal energy. The low-temperature expansion is of course only asymptotic, but the high-temperature limit is convergent, and simple enough to even determine its convergence radius. In Section 5, we calculate the analogous series expansions of the other thermodynamic variables, such as heat capacities and entropy/energy per tachyon. The equations of state are rather different from those of a photonic or subluminal massive Bose gas, due to the negative mass square and the absence of a chemical potential, and so we give a complete, self-contained derivation.

In Section 6, the Conclusion, bounds on absorption and emission rates for tachyon radiation in hydrogenic systems, including Rydberg transitions, are derived. The 
estimates are based on a tachyon mass of $m_{\mathrm{t}} \approx m_{\mathrm{e}} / 238 \approx 2.15 \mathrm{keV} / c^{2}$, and a tachyonic fine structure constant $\beta \approx 1.0 \times 10^{-13} \approx 0.66 \alpha^{6}$, both obtained in Refs. [25,28]. (In these papers, we studied level shifts in hydrogen-like systems induced by the static potential of this field theory, and compared the shifts to the discrepancy between Lamb shift measurements and QED calculations.) The spectral energy density of the cosmic tachyon radiation deviates from the Rayleigh-Jeans low-frequency limit, and we will demonstrate, by comparing tachyonic and photonic absorption rates, that the tachyon background overpowers the photon radiation for frequencies below $7.3 \mathrm{MHz}$.

\section{The wave equation for tachyons and its coupling to subluminal matter}

We use locally geodesic coordinates, i.e., Minkowski space, $\eta_{\mu v}=\operatorname{diag}\left(-c^{2}, 1,1,1\right)$. The Proca equation with negative mass square,

$$
F_{, \beta}^{\alpha \beta}-\mu^{2} A^{\alpha}=c^{-1} j^{\alpha},
$$

( $\mu=m_{\mathrm{t}} c / \hbar>0$ in our notation) can be derived from the action

$$
S=\int\left(L_{A}+L_{\text {int }}\right) \mathrm{d} t \mathrm{~d} x, \quad L_{A}=-\frac{1}{4} F_{\alpha \beta} F^{\alpha \beta}+\frac{1}{2} \mu^{2} A_{\alpha} A^{\alpha}, \quad L_{\text {int }}=\frac{1}{c} j^{\alpha} A_{\alpha},
$$

and the energy-momentum tensor for the Proca field reads as

$$
T_{\mu}^{v}=-F_{\alpha \mu} F^{\alpha v}+\mu^{2} A_{\mu} A^{v}+\delta_{\mu}^{v}\left(\frac{1}{4} F_{\alpha \beta} F^{\alpha \beta}-\frac{1}{2} \mu^{2} A_{\alpha} A^{\alpha}\right) .
$$

Eq. (2.1) is easily seen to be equivalent to

$$
\left(\square+\mu^{2}\right) A_{\alpha}=-c^{-1} j_{\alpha}, \quad A_{, \mu}^{\mu}=0,
$$

with the d'Alembertian $\square:=\eta^{\mu v} \partial_{\mu} \partial_{v}$; the Lorentz condition is a consequence of current conservation, $j_{, \mu}^{\mu}=0$. Tachyonic $E$ and $B$-fields are related to the vector potential by

$$
\begin{aligned}
& E_{i}=c^{-1} F_{i 0}, \quad F_{i j}=\varepsilon_{i j k} B^{k}, \quad F_{\alpha \beta}=A_{\beta, \alpha}-A_{\alpha, \beta}, \\
& B^{k}=(1 / 2) \varepsilon^{k i j} F_{i j}=\operatorname{rot} \mathbf{A} .
\end{aligned}
$$

The field equations (2.1) or (2.4) can be written as

$$
\begin{aligned}
& \operatorname{div} \mathbf{B}=0, \quad \operatorname{rot} \mathbf{E}+c^{-1} \partial \mathbf{B} / \partial t=0, \\
& \operatorname{div} \mathbf{E}=\rho-c^{-1} \mu^{2} A_{0}, \quad \operatorname{rot} \mathbf{B}-c^{-1} \partial \mathbf{E} / \partial t=c^{-1} \mathbf{j}+\mu^{2} \mathbf{A},
\end{aligned}
$$

where we identified $j^{\mu}=(\rho, \mathbf{j})$. Evidently, the vector potential is completely determined by the current and the $\mathbf{E}$ and $\mathbf{B}$ fields. We may substitute $F_{\alpha \beta} F^{\alpha \beta}=-2\left(\mathbf{E}^{2}-\mathbf{B}^{2}\right)$ into the Lagrangian (2.2), and the classical energy density and the Poynting vector are readily found as

$$
\begin{aligned}
& \rho_{E}=T_{0}^{0}=(1 / 2)\left(\mathbf{E}^{2}+\mathbf{B}^{2}\right)-\left(\mu^{2} / 2\right)\left(c^{-2} A_{0} A_{0}+\mathbf{A}^{2}\right), \\
& \mathbf{S}=T_{0}^{m}=c \mathbf{E} \times \mathbf{B}+\mu^{2} A_{0} \mathbf{A} .
\end{aligned}
$$


The spectral elementary waves of the free wave equation (2.4) are

$$
\begin{aligned}
& A_{\alpha}(t, \mathbf{x})=\hat{A}_{\alpha}(\mathbf{k}) \exp (\mathrm{i}(\mathbf{k} \mathbf{x}-\omega t))+\text { c.c. }, \\
& \hat{A}_{\alpha}(\mathbf{k}):=\varepsilon_{\alpha}^{\mathrm{T} 1} \hat{a}^{\mathrm{T} 1}(\mathbf{k})+\varepsilon_{\alpha}^{\mathrm{T} 2} \hat{a}^{\mathrm{T} 2}(\mathbf{k})+\varepsilon_{\alpha}^{\mathrm{L}} \hat{a}^{\mathrm{L}}(\mathbf{k}), \\
& \varepsilon_{\alpha}^{\mathrm{T} i}(\mathbf{k}):=\left(0, \mathbf{e}^{i}\right), \quad \varepsilon_{\alpha}^{\mathrm{L}}:=\left(-\frac{c|\mathbf{k}|}{\mu}, \frac{\omega}{\mu c} \frac{\mathbf{k}}{|\mathbf{k}|}\right), \quad|\mathbf{k}|=c^{-1} \sqrt{\omega^{2}+\mu^{2} c^{2}} .
\end{aligned}
$$

The 3 -vectors $\mathbf{e}^{i}, i=1,2$, are conveniently chosen Euclidean unit vectors transversal to the direction of propagation, so that $\mathbf{e}^{i} \cdot \mathbf{k}=0$ and $\mathbf{e}^{1} \cdot \mathbf{e}^{2}=0$. The space component of the longitudinal polarization vector $\varepsilon_{\alpha}^{\mathrm{L}}$ is of course proportional to $\mathbf{k}$, the time component is chosen in a way that the Lorentz condition, $c^{-2} \hat{A}_{0} \omega+\hat{\mathbf{A}} \cdot \mathbf{k}=0$, is satisfied. The normalization $\varepsilon_{\alpha}^{\mathrm{L}} \varepsilon^{\mathrm{L} \alpha}=-1$ is adopted, so that $\varepsilon_{\alpha}^{\mathrm{L}}$ stays well defined for $\omega=0$. The Fourier amplitudes $\hat{a}^{\mathrm{T} i, \mathrm{~L}}(\mathbf{k})$ are arbitrary complex numbers. Transversal and longitudinal components are denoted by superscripts $\mathrm{T}$ (or $\mathrm{T} i$ for $\mathrm{e}^{i}$-polarization) and $\mathrm{L}$. We may write the transversal and longitudinal components of $A_{\alpha}$ as

$$
\begin{aligned}
& A_{\alpha}^{\mathrm{T} i}=\varepsilon_{\alpha}^{\mathrm{T} i}\left(a^{\mathrm{T} i}+a^{\mathrm{T} i *}\right), \quad A_{\alpha}^{\mathrm{L}}=\varepsilon_{\alpha}^{\mathrm{L}}\left(a^{\mathrm{L}}+a^{\mathrm{L} *}\right), \\
& a^{\mathrm{T} i, \mathrm{~L}}(\mathbf{k} ; t, \mathbf{x}):=\hat{a}^{\mathrm{T} i, \mathrm{~L}}(\mathbf{k}) \exp (\mathrm{i}(\mathbf{k} \mathbf{x}-\omega t)),
\end{aligned}
$$

respectively, and the corresponding components of the $\mathbf{E}$ and $\mathbf{B}$-fields are given by

$$
\begin{aligned}
& \mathbf{E}^{\mathrm{T} i}=\mathrm{i}(\omega / c) \mathbf{e}^{i}\left(a^{\mathrm{T} i}-a^{\mathrm{T} i *}\right), \quad \mathbf{B}^{\mathrm{T} i}=\mathrm{i}\left(\mathbf{k} \times \mathbf{e}^{i}\right)\left(a^{\mathrm{T} i}-a^{\mathrm{T} i *}\right), \\
& \mathbf{E}^{\mathrm{L}}=\frac{\mu}{\mathrm{i}} \frac{\mathbf{k}}{|\mathbf{k}|}\left(a^{\mathrm{L}}-a^{\mathrm{L} *}\right), \quad \mathbf{B}^{\mathrm{L}}=0 .
\end{aligned}
$$

The energy density, defined by (2.7), reads for each of these modes

$$
\begin{aligned}
& \rho_{E}^{\mathrm{T} i}=(1 / 2)\left(\left(a^{\mathrm{T} i}-a^{\mathrm{T} i *}\right)^{2}\left(\mu^{2}-2 \mathbf{k}^{2}\right)-\mu^{2}\left(a^{\mathrm{T} i}+a^{\mathrm{T} i *}\right)^{2}\right), \\
& \rho_{E}^{\mathrm{L}}=(1 / 2)\left(\left(a^{\mathrm{L}}+a^{\mathrm{L} *}\right)^{2}\left(\mu^{2}-2 \mathbf{k}^{2}\right)-\mu^{2}\left(a^{\mathrm{L}}-a^{\mathrm{L} *}\right)^{2}\right),
\end{aligned}
$$

and the energy flux vector (2.8), likewise defined for every single transversal and longitudinal field component, is readily calculated as

$$
\mathbf{S}^{\mathrm{T} i}=-\omega \mathbf{k}\left(a^{\mathrm{T} i}-a^{\mathrm{T} i *}\right)^{2}, \quad \mathbf{S}^{\mathrm{L}}=-\omega \mathbf{k}\left(a^{\mathrm{L}}+a^{\mathrm{L} *}\right)^{2} .
$$

When averaged over a period of $2 \pi / \omega$, energy density and flux read

$$
\left\langle\rho_{E}^{\mathrm{T} i}\right\rangle=\frac{2}{c^{2}}\left|\hat{a}^{\mathrm{T} i}\right|^{2} \omega^{2}, \quad\left\langle\rho_{E}^{\mathrm{L}}\right\rangle=-\frac{2}{c^{2}}\left|\hat{a}^{\mathrm{L}}\right|^{2} \omega^{2}, \quad\left\langle\mathbf{S}^{\mathrm{T} i, \mathrm{~L}}\right\rangle=c^{2} \frac{\mathbf{k}}{\omega}\left\langle\rho_{E}^{\mathrm{T} i, \mathrm{~L}}\right\rangle .
$$

The energy density of the transversal modes $\rho_{E}^{\mathrm{T} i}$ (or its time average) is, as a function of frequency, bounded from below. $\left\langle\rho_{E}^{\mathrm{L}}\right\rangle$ is bounded from above, so that we define the energy density of the longitudinal modes as well as their Poynting vector with opposite sign: $\rho_{E}^{\mathrm{L}}:=-T_{0}^{0}$ and $\mathbf{S}^{\mathrm{L}}:=-T_{0}^{m}$, with $T_{0}^{\mu}$ as in (2.7) and (2.8), which amounts to replace 
$\left(\rho_{E}^{\mathrm{L}}, \mathbf{S}^{\mathrm{L}}\right)$ by $\left(-\rho_{E}^{\mathrm{L}},-\mathbf{S}^{\mathrm{L}}\right)$ in $(2.13)-(2.15)$. The energy-momentum tensor of the longitudinal modes is $-T_{\mu}^{v}$, cf. (2.3). Transversal modes in the galaxy frame (i.e., the comoving Robertson-Walker frame in which the galaxies have constant space coordinates) will usually appear in Minkowskian rest frames (of observers moving relative to the galaxy grid) with a longitudinal component. To illustrate this, we consider a transversal spectral mode, cf. (2.9), $A_{0}=0, \mathbf{A} \cdot \mathbf{k}=0$, in the galaxy frame $(t, \mathbf{x})$, which we assume here static and Minkowskian for technical convenience, and apply a Lorentz boost along the $x$-axis, corresponding to the geodesic rest frame $\left(t^{\prime}, \mathbf{x}^{\prime}\right)$ of an observer moving in the static galaxy grid,

$$
\begin{aligned}
& A_{0}^{\prime}=\gamma v A_{1}, \quad A_{1}^{\prime}=\gamma A_{1}, \quad A_{2,3}^{\prime}=A_{2,3}, \\
& t=\gamma\left(t^{\prime}+v c^{-2} x^{\prime}\right), \quad x=\gamma\left(x^{\prime}+v t^{\prime}\right), \quad \gamma:=\left(1-v^{2} / c^{2}\right)^{-1 / 2} .
\end{aligned}
$$

Frequencies and wave vectors relate as

$$
\omega^{\prime}=\gamma\left(\omega-v k_{1}\right), \quad k_{1}^{\prime}=\gamma\left(k_{1}-v c^{-2} \omega\right), \quad k_{2,3}^{\prime}=k_{2,3}, \quad\left|\mathbf{k}^{\prime}\right|=\sqrt{\omega^{\prime 2} / c^{2}+\mu^{2}} .
$$

Accordingly, if a Lorentz boost is applied to a transversally polarized wave, there is always a longitudinal component generated, unless the velocity in the boost is orthogonal to the polarization. Moreover, a Lorentz boost can always be found so that $\omega^{\prime}=: E^{\prime} / \hbar$ is zero or an arbitrary negative number. In the galactic reference frame, $\omega$ is by definition positive. As Lorentz boosts mix transversal and longitudinal components, a universal reference frame is not only necessary for the causality interpretation of superluminal signals $[18,19,21,26]$, but also to unambiguously define a positive energy for transversal and longitudinal tachyons.

We specify the current in (2.1) by assuming that subluminal particles are coupled to the tachyonic vector potential in the same way as electric charges to the electromagnetic field, the electric charge being replaced by a tachyonic charge. The Lagrangian of a subluminal particle (with rest mass $m$ and tachyonic charge $q$ ) coupled to the tachyon potential is

$$
L^{(3)}:=-m c^{2} \sqrt{1-\mathbf{v}^{2} / c^{2}}+q c^{-1} A_{0}+q c^{-1} \mathbf{A} \mathbf{v},
$$

the action can be written as

$$
S=\int L^{(3)} \mathrm{d} t=c^{-1} \int L \mathrm{~d} s, \quad L:=-m c^{2} \sqrt{-\eta_{\mu \nu} \dot{x}^{\mu} \dot{x}^{\nu}}+q A_{\mu} \dot{x}^{\mu},
$$

resulting in the Hamilton-Jacobi equation

$$
\eta^{\mu v}\left(S_{, \mu}-c^{-1} q A_{\mu}\right)\left(S_{, v}-c^{-1} q A_{v}\right)=-m^{2} c^{2},
$$

and the Newton equations

$$
\frac{\mathrm{d}}{\mathrm{d} t} \frac{m \mathbf{v}}{\sqrt{1-\mathbf{v}^{2} / c^{2}}}=q \mathbf{E}+\frac{q}{c} \mathbf{v} \times \mathbf{B},
$$


with a tachyonic Lorentz force. To fix the signs, the interaction terms in (2.19) are incorporated into the action (2.2) by

$$
\begin{aligned}
& S_{\text {int }}=\int L_{\text {int }} \mathrm{d} t \mathrm{~d} \mathbf{x}=c^{-1} \int\left(q A_{0}+q \mathbf{A} \mathbf{v}\right) \mathrm{d} t=c^{-1} \int A_{\alpha} j^{\alpha} \mathrm{d} t \mathrm{~d} \mathbf{x}, \\
& j^{0}=\rho=q \delta(\mathbf{x}-\mathbf{x}(t)), \quad \mathbf{j}=q \mathbf{v} \delta(\mathbf{x}-\mathbf{x}(t)) ;
\end{aligned}
$$

in this way the current in (2.2) is identified. As for the coupling of the Dirac equation,

$$
\begin{aligned}
& \gamma^{\alpha} \nabla_{\alpha}^{A} \psi+(m c / \hbar) \psi=0, \quad \nabla_{\alpha}^{A}:=\partial_{\alpha}-\mathrm{i} q /(\hbar c) A_{\alpha}, \\
& S_{\psi}=\int L_{\psi} \mathrm{d} t \mathrm{~d} x, \quad L_{\psi}=\frac{\hbar}{\mathrm{i}}\left(\frac{1}{2} \bar{\psi} \gamma^{\alpha} \nabla_{\alpha}^{A} \psi-\frac{1}{2}\left(\nabla_{\alpha}^{A *} \bar{\psi}\right) \gamma^{\alpha} \psi+\frac{m c}{\hbar} \psi \bar{\psi}\right),
\end{aligned}
$$

with $\bar{\psi}:=\psi^{t *} \gamma^{0}$. The current reads $j^{\mu}=-q \bar{\psi} \gamma^{\mu} \psi$, and if we use the set of $\gamma$-matrices

$$
\gamma^{0}=\frac{1}{\mathrm{i} c}\left(\begin{array}{cc}
\mathrm{id} & 0 \\
0 & -\mathrm{id}
\end{array}\right), \quad \gamma^{k}=\left(\begin{array}{cc}
0 & -\mathrm{i} \sigma_{k} \\
\mathrm{i} \sigma_{k} & 0
\end{array}\right),
$$

with Pauli matrices $\sigma_{k}$, we obtain the charge density $\rho=j^{0}=q \psi^{t *} \psi$. The sign of $L_{\psi}$ in (2.24) is chosen in a way that the current can be identified with $j^{\mu}$ in (2.2) when $L_{\psi}$ is substituted for $L_{\text {int }}$. The non-relativistic limit of (2.23), the Pauli equation, is discussed in [28]. Finally, the non-relativistic limit of (2.20) is readily found as

$$
S_{, 0}-c^{-1} q A_{0}+(2 m)^{-1}\left(S_{, i}-c^{-1} q A_{i}\right)\left(S_{, i}-c^{-1} q A_{i}\right)=0,
$$

and the corresponding Schrödinger equation,

$$
\frac{1}{\mathrm{i}} \partial_{t}^{A} \psi=\frac{\hbar}{2 m} \nabla^{A} \nabla^{A} \psi, \quad \partial_{t}^{A}:=\partial_{t}-\mathrm{i} q /(\hbar c) A_{0}, \quad \nabla^{A}:=\nabla-\mathrm{i} q /(\hbar c) \mathbf{A},
$$

can be derived from the Lagrangian

$$
L=-\frac{\hbar}{2 \mathrm{i}}\left(\psi^{*} \partial_{t}^{A} \psi-\psi \partial_{t}^{A *} \psi^{*}\right)-\frac{\hbar^{2}}{2 m} \nabla^{A} \psi \nabla^{A *} \psi^{*},
$$

which is to be substituted for $L_{\text {int }}$ in (2.2), so that the tachyonic charge density is identified as $j^{0}=\rho=q \psi^{*} \psi$.

\section{Emission and absorption of tachyons in atomic transitions}

The inversion of the wave equation (2.4) can formally be effected by

$$
\begin{aligned}
& A_{\alpha}(x)=\int_{R^{4}} G\left(x-x^{\prime}\right) j_{\alpha}\left(x^{\prime}\right) \mathrm{d} x^{\prime}, \\
& \left(\square+\mu^{2}\right) G(t, \mathbf{x})=-c^{-1} \delta(t) \delta(\mathbf{x}) ;
\end{aligned}
$$

the Lorentz condition is evidently satisfied by this ansatz. In the case of photons or subluminal particles $\left(\mu^{2} \leqslant 0\right)$, one would choose for $G$ the retarded Green function, 
supported on the forward light cone. The classical Green function for tachyons is supported outside the light cone (at least as long as we do not consider curvature effects) because tachyons cannot move below the speed of light, and hence they cannot connect events in the interior of the cone. The complete solution of (3.2) is readily obtained by analytic continuation, $\mu \rightarrow \pm \mathrm{i} \mu$, of the subluminal Green functions (retarded, advanced, Feynman and Dyson propagators, listed in Ref. [29]). The only linear combination of these continuations vanishing inside the cone is

$$
G(t, \mathbf{x})=\frac{1}{4 \pi} \delta\left(r^{2}-c^{2} t^{2}\right)-\frac{\mu}{8 \pi} \theta\left(r^{2}-c^{2} t^{2}\right) \frac{J_{1}\left(\mu \sqrt{r^{2}-c^{2} t^{2}}\right)}{\sqrt{r^{2}-c^{2} t^{2}}} .
$$

A detailed discussion of Green functions of the Proca equation, in Robertson-Walker cosmology, will be given elsewhere. This distribution admits a very handy Fourier transform,

$$
\int_{-\infty}^{+\infty} G(t, \mathbf{x}) \mathrm{e}^{-\mathrm{i} \omega t} \mathrm{~d} t=\frac{1}{4 \pi c} \frac{1}{r} \cos (k r),
$$

with $k=\sqrt{\omega^{2} / c^{2}+\mu^{2}}$.

We study a periodic time dependence of the current and the fields in (2.4) and (2.6), writing, cf. (2.9),

$$
\rho(\mathbf{x}, t):=\rho(\mathbf{x}, \omega) \mathrm{e}^{-\mathrm{i} \omega t}+\text { c.c., } \quad \mathbf{A}(\mathbf{x}, t):=\mathbf{A}(\mathbf{x}, \omega) \mathrm{e}^{-\mathrm{i} \omega t}+\text { c.c. },
$$

with complex $\rho(\mathbf{x}, \omega), \mathbf{A}(\mathbf{x}, \omega)$, and analogously for the other fields in (2.6). (In the following, we will mostly drop the $\omega$-argument.) The Fourier components of the $E$ and $B$-fields are found as

$$
\mathbf{B}(\mathbf{x})=\nabla \times \mathbf{A}(\mathbf{x}), \quad \mathbf{E}(\mathbf{x})=\mathrm{ic} \omega^{-1}\left(\nabla(\nabla \cdot \mathbf{A}(\mathbf{x}))+\left(\omega^{2} / c^{2}\right) \mathbf{A}(\mathbf{x})\right) .
$$

Continuity equation and Lorentz condition read

$$
\mathrm{i} \omega \rho(\mathbf{x})=\nabla \cdot \mathbf{j}(\mathbf{x}), \quad \mathrm{i} \omega A_{0}(\mathbf{x})=-c^{2} \nabla \cdot \mathbf{A}(\mathbf{x}),
$$

and the Maxwell equations in Fourier space are obtained by substituting $\partial / \partial \tau \rightarrow-\mathrm{i} \omega$ into (2.6).

By virtue of (3.1) and (3.4), we find $\mathbf{A}(\mathbf{x}, \omega)=\mathbf{A}^{+}+\mathbf{A}^{-}$, with

$$
\mathbf{A}^{ \pm}(\mathbf{x}, \omega):=\frac{1}{8 \pi c} \int \mathrm{d} \mathbf{x}^{\prime} \mathbf{j}\left(\mathbf{x}^{\prime}, \omega\right) \frac{\exp \left( \pm \mathrm{ik}\left|\mathbf{x}-\mathbf{x}^{\prime}\right|\right)}{\left|\mathbf{x}-\mathbf{x}^{\prime}\right|} ;
$$

the time components $A_{0}^{ \pm}(\mathbf{x}, \omega)$ follow from (3.8) via the substitution $\mathbf{j}\left(\mathbf{x}^{\prime}, \omega\right) \rightarrow$ $-c^{2} \rho\left(\mathbf{x}^{\prime}, \omega\right)$. Using $\left|\mathbf{x}-\mathbf{x}^{\prime}\right|=r-\mathbf{n} \cdot \mathbf{x}^{\prime}+\mathrm{O}(1 / r), \mathbf{n}:=\mathbf{x} / r$, we obtain, asymptotically,

$$
\mathbf{A}^{ \pm}(\mathbf{x}) \sim \frac{1}{8 \pi c} \frac{\mathrm{e}^{ \pm \mathrm{i} k r}}{r} \mathbf{J}^{ \pm}, \quad \mathbf{J}^{ \pm}:=\int \mathrm{d} \mathbf{x}^{\prime} \mathbf{j}\left(\mathbf{x}^{\prime}\right) \exp \left(\mp \mathrm{ikn} \cdot \mathbf{x}^{\prime}\right) .
$$

We will give in some detail the derivation of the transition rates effected by transversal tachyons, and afterwards point out the minor modifications for longitudinal transitions. The transversal component, $\mathbf{A}^{\mathrm{T} \pm}(\mathbf{x})$, is found in leading order by replacing $\mathbf{J}^{ \pm}$by $\mathbf{J}^{\mathrm{T} \pm}:=\mathbf{J}^{ \pm}-\mathbf{n}\left(\mathbf{n} \cdot \mathbf{J}^{ \pm}\right)$; moreover, via (3.7), $\mathbf{A}_{0}^{\mathrm{T} \pm}=\mathrm{O}\left(1 / r^{2}\right)$. The advanced component 
of the wave field can be converted into a retarded one, $\mathbf{A}^{\mathrm{T}-} \rightarrow \mathbf{A}^{\mathrm{T}+}$, by a cosmic absorber [13,14]. Apart from the causality interpretation, the definition of tachyonic energy, and the cosmic time scaling of the tachyon mass, cf. the beginning of Section 4, the cosmological reference frame is here once more required to turn advanced wave fields into retarded ones. Unlike in electrodynamics or subluminal field theories, there does not exist a proper retarded or advanced Green function for tachyons, as the outside of the light cone is a connected domain; the time symmetry is broken by an absorber, by the permeability of the cosmic ether [20,30-34], which is straightforward to define in an absolute space-time. An account on superluminal wave propagation in the ether will be given elsewhere; at his point it is sufficient to assume that the ether breaks the time symmetry of the wave fields by converting advanced modes into retarded ones. The retarded and advanced wave fields $2 \mathbf{A}_{\mu}^{+/-}$as defined in (3.8) are exact solutions of the field equations (2.1) or (2.4), because $\left(\Delta+k^{2}\right)\left(r^{-1} \sin (k r)\right) \equiv 0$. The Fourier components of the wave fields generated by the current are thus

$$
\begin{aligned}
& \mathbf{A}^{\mathrm{ret}}(\mathbf{x}):=2 \mathbf{A}^{\mathrm{T}+} \sim \frac{1}{4 \pi c} \frac{\mathrm{e}^{\mathrm{i} k r}}{r} \mathbf{J}^{\mathrm{T}+}, \\
& \mathbf{E}^{\mathrm{ret}}(\mathbf{x}) \sim \frac{\mathrm{i}}{4 \pi c} \frac{\omega}{c} \frac{\mathrm{e}^{\mathrm{i} k r}}{r} \mathbf{J}^{\mathrm{T}+}, \quad \mathbf{B}^{\mathrm{ret}}(\mathbf{x}) \sim \frac{\mathrm{i}}{4 \pi c} k \frac{\mathrm{e}^{\mathrm{i} k r}}{r}\left(\mathbf{n} \times \mathbf{J}^{\mathrm{T}+}\right) .
\end{aligned}
$$

Energy density and flux of these modes, time-averaged over a period of $2 \pi / \omega$, read as

$$
\left\langle\rho_{E}\right\rangle \sim \frac{2}{(4 \pi c)^{2}} \frac{1}{r^{2}} \frac{\omega^{2}}{c^{2}}\left|\mathbf{J}^{\mathrm{T}+}\right|^{2}, \quad\langle\mathbf{S}\rangle \sim c^{2} k \omega^{-1}\left\langle\rho_{E}\right\rangle \mathbf{n} .
$$

The following semiclassical analysis of spontaneous emission and induced absorption of tachyons is kept close to the photonic counterpart, e.g., Refs. [35,36], it will be concise, mainly focussing on technical modifications due to the tachyon mass. We start by defining

$$
\begin{aligned}
& \mathbf{J}^{(\mathrm{T}) \mathrm{d}}:=\int \mathrm{d} \mathbf{x} \mathbf{j}^{(\mathrm{T})}(\mathbf{x})=-\mathrm{i} \omega \mathbf{d}^{(\mathrm{T})}, \quad \mathbf{j}^{\mathrm{T}}\left(\mathbf{x}^{\prime}\right):=\mathbf{j}\left(\mathbf{x}^{\prime}\right)-\mathbf{n}\left(\mathbf{n} \cdot \mathbf{j}\left(\mathbf{x}^{\prime}\right)\right), \\
& \mathbf{d}:=\int \mathbf{r} \rho(\mathbf{x}) \mathrm{d} \mathbf{x}, \quad \mathbf{d}^{\mathrm{T}}:=\mathbf{d}-\mathbf{n}(\mathbf{n} \cdot \mathbf{d}),
\end{aligned}
$$

where we again used (3.7). The dipole approximation of (3.10) and (3.11) is obtained by dropping the exponential in $\mathbf{J}^{+}$, which means to substitute $\mathbf{J}^{\mathrm{T}+} \rightarrow \mathbf{J}^{\mathrm{Td}}$. (We then write $\rho_{E}^{\mathrm{d}}$ and $\mathbf{S}^{\mathrm{d}}$, respectively.) We so obtain for the energy radiated per unit time

$$
\int\left\langle\mathbf{S}^{\mathrm{d}} \cdot \mathbf{n}\right\rangle r^{2} \mathrm{~d} \Omega \sim \frac{16 \pi}{3} \frac{k \omega^{3}}{(4 \pi c)^{2}}|\mathbf{d}|^{2},
$$

where we made use of

$$
\int\left|\mathbf{J}^{\mathrm{Td}}\right|^{2} \mathrm{~d} \Omega=\left|\mathbf{J}^{\mathrm{d}}\right|^{2} \int \sin ^{2} \theta \mathrm{d} \Omega=(8 \pi / 3)\left|\mathbf{J}^{\mathrm{d}}\right|^{2},
$$

$\mathrm{d} \Omega=\sin \theta \mathrm{d} \theta \mathrm{d} \varphi$, and (3.12). What remains is to relate the dipole to the quantum current. 
In the Schrödinger equation (2.27), we neglect $A_{0}$, see after (3.9), and consider only the interaction term linear in $\mathbf{A}$, so that

$$
\mathrm{i} \hbar \partial_{\mathrm{t}} \psi=\left(-\frac{\hbar^{2}}{2 m} \Delta-\frac{Z e^{2}}{4 \pi} \frac{1}{r}+\frac{\mathrm{i} q \hbar}{m c} \mathbf{A} \cdot \nabla\right) \psi .
$$

We have here included the Coulomb potential of a nucleus of electric charge $-Z e$; the tachyonic vector potential is treated perturbatively. The quantum analog of the Fourier components $\rho(\mathbf{x})$ and $\mathbf{j}(\mathbf{x})$ is

$$
\rho(\mathbf{x})=q u_{\mathrm{f}}^{*} u_{\mathrm{i}}, \quad \mathbf{j}(\mathbf{x})=\frac{q \hbar}{2 \mathrm{i} m}\left(u_{\mathrm{f}}^{*} \nabla u_{\mathrm{i}}-\left(\nabla u_{\mathrm{f}}^{*}\right) u_{\mathrm{i}}\right),
$$

where $u_{\mathrm{i}, \mathrm{f}}$ are the time separated, normalized wave functions of the initial and final states, respectively. In dipole approximation, the probability per unit time for the spontaneous emission of a transversal tachyon (of frequency $\omega=\omega_{\mathrm{i}}-\omega_{\mathrm{f}}>0$, via a transition $\left.u_{\mathrm{i}} \rightarrow u_{\mathrm{f}}\right)$ is thus, cf. (3.13), (3.12) and (3.16),

$$
w_{\text {sp.em. }}^{\mathrm{d}}=\frac{1}{\hbar \omega} \int\left\langle\mathbf{S}^{\mathrm{d}} \cdot \mathbf{n}\right\rangle r^{2} \mathrm{~d} \Omega \sim \frac{4}{3} \frac{k \omega^{2}}{c^{2} \hbar} \frac{q^{2}}{4 \pi}\left|\int u_{\mathrm{f}}^{*} \mathbf{r} u_{\mathrm{i}} \mathrm{d} \mathbf{x}\right|^{2}=: A_{\mathrm{if}}^{\mathrm{d}},
$$

with $k:=\sqrt{\omega^{2} / c^{2}+\mu^{2}} ; A_{\text {if }}^{\mathrm{d}}$ is the Einstein $A$-coefficient in dipole approximation. If we refrain from the dipole approximation, we find, instead of (3.13), for the energy radiated into the solid angle per unit time,

$$
\langle\mathbf{S} \cdot \mathbf{n}\rangle r^{2} \mathrm{~d} \Omega \sim \frac{2 \mathrm{~d} \Omega}{(4 \pi c)^{2}} k \omega\left|\mathbf{J}^{\mathrm{T}+}\right|^{2} .
$$

We substitute into $\mathbf{J}^{\mathrm{T}+}$ the transversal component of the quantum current (3.16). Dividing by $\hbar \omega$, we find the probability per unit time for the spontaneous emission of a transversal tachyon with wave vector $\mathbf{k}=k \mathbf{n}(\theta, \varphi)$ into the solid angle,

$$
\mathrm{d} w_{\text {sp.em. }}^{\text {unpol. }} \sim 2 \frac{\hbar k}{m^{2} c^{2}} \frac{q^{2}}{4 \pi}\left|\int u_{\mathrm{f}}^{*} \exp \left(-\mathrm{i} \mathbf{k} \mathbf{x}^{\prime}\right) \nabla^{\mathrm{T}} u_{\mathrm{i}} \mathrm{d} \mathbf{x}^{\prime}\right|^{2} \frac{\mathrm{d} \Omega}{4 \pi},
$$

$k$ is defined with the transition frequency like in the dipole approximation, and $\nabla^{\mathrm{T}}:=\nabla-$ $\mathbf{n}(\mathbf{n} \cdot \nabla)$ is the transversal component of the gradient, so that (3.19) is a polarization average. If we focus on radiation of a given polarization $\mathbf{e}$, we just have to replace in (3.19) $\nabla^{\mathrm{T}}$ by $\mathbf{e} \cdot \nabla$, to find the probability for the emission of a tachyon $(\mathbf{e}, \mathbf{k})$ via a spontaneous transition $u_{\mathrm{i}} \rightarrow u_{\mathrm{f}}$,

$$
\mathrm{d} w_{\text {sp.em. }} \sim A_{\text {if }} \mathrm{d} \Omega, \quad A_{\text {if }}(\mathbf{k}, \mathbf{e}):=\frac{1}{2 \pi} \frac{\hbar k}{m^{2} c^{2}} \frac{q^{2}}{4 \pi}\left|\int u_{\mathrm{f}}^{*} \exp \left(-\mathrm{ik} \mathbf{x}^{\prime}\right) \mathbf{e} \cdot \nabla u_{\mathrm{i}} \mathrm{d} \mathbf{x}^{\prime}\right|^{2} .
$$

Next, we will relate this to induced radiation processes. To this end, we start with the Schrödinger equation (3.15), and consider a transversal plane wave as in (2.9). (We drop the polarization index $\mathrm{i}$ for convenience; $\mathbf{e}$ is an arbitrary unit vector orthogonal to k.) The first-order transition amplitude of this periodic perturbation reads, cf. [35],

$$
T_{\mathrm{if}}^{ \pm}(\omega) \sim \frac{1}{\hbar} \frac{1-\exp \left(\mathrm{i}\left(\omega_{\mathrm{fi}} \mp \omega\right) t\right)}{\omega_{\mathrm{fi}} \mp \omega}\left\langle f\left|H_{\mathrm{int}}^{ \pm}\right| \mathrm{i}\right\rangle,
$$




$$
\left\langle f\left|H_{\mathrm{int}}^{ \pm}\right| \mathrm{i}\right\rangle=\frac{\mathrm{i} q \hbar}{m c} \hat{a}^{T(*)} \int u_{\mathrm{f}}^{*} \exp ( \pm \mathrm{ikx}) \mathbf{e} \cdot \nabla u_{\mathrm{i}} \mathrm{d} \mathbf{x},
$$

where $u_{\mathrm{i}, \mathrm{f}}$ are the initial and final states of the unperturbed Coulomb problem (3.15), and $\omega_{\mathrm{fi}}:=\omega_{\mathrm{f}}-\omega_{\mathrm{i}}$ is the transition frequency. The complex conjugation of $\hat{a}^{\mathrm{T}}$ refers to $H_{\text {int }}^{-}$only. If $\omega_{\text {fi }}>0$, then $\mathrm{T}_{\text {if }}^{+}(\omega)$ applies (absorption), otherwise $\mathrm{T}_{\text {if }}^{-}(\omega)$ (emission). In either case, the absorbed or emitted tachyon has polarization $\mathbf{e}$ and wave vector $\mathbf{k}$.

We may identify, cf. (2.15),

$$
\frac{2}{c^{2}}\left|\hat{a}^{\mathrm{T}}\right|^{2} \omega^{2}=\frac{1}{2} \rho_{E}(\omega) \mathrm{d} \omega,
$$

where $\rho_{E}(\omega)$ is the spectral energy density of the transversal modes, cf. (3.32) and (4.3). The superscript $\mathrm{T}$ in (3.23) refers to one of the two independent transversal polarizations $\mathrm{Ti}$, therefore the factor of one-half. The $\omega$-integration of $\left|\mathrm{T}_{\text {if }}^{ \pm}(\omega)\right|^{2}$ can readily be carried out by steepest descent, and the probability per unit time for the emission/absorption of a tachyon $(\mathbf{e}, \mathbf{k})$ through the solid angle centered at $\mathbf{k}$, in an induced transition $u_{\mathrm{i}} \rightarrow u_{\mathrm{f}}$, is thus given by

$$
\begin{aligned}
& \mathrm{d} w_{\text {ind.em. }} \sim \frac{1}{t}\left|\mathrm{~T}_{\text {if }}^{-}\right|^{2} \frac{\mathrm{d} \Omega}{4 \pi} \sim B_{\text {if }}(\mathbf{k}, \mathbf{e}) \rho_{E}(\omega) \mathrm{d} \Omega, \\
& \mathrm{d} w_{\text {ind.abs. }} \sim \frac{1}{t}\left|\mathrm{~T}_{\text {if }}^{+}\right|^{2} \frac{\mathrm{d} \Omega}{4 \pi} \sim B_{\text {if }}(-\mathbf{k}, \mathbf{e}) \rho_{E}(\omega) \mathrm{d} \Omega, \\
& B_{\text {if }}(\mathbf{k}, \mathbf{e}):=\frac{\pi}{2} \frac{1}{m^{2} \omega^{2}} \frac{q^{2}}{4 \pi}\left|\int u_{\mathrm{f}}^{*} \exp (-\mathrm{ikx}) \mathbf{e} \cdot \nabla u_{\mathrm{i}} \mathrm{d} \mathbf{x}\right|^{2},
\end{aligned}
$$

with the transition frequency $\omega=\left|\omega_{\mathrm{i}}-\omega_{\mathrm{f}}\right|$. (The $\left|\mathrm{T}_{\text {if }}^{ \pm}\right|^{2}$ are $\omega$-integrated.) The Einstein coefficients admit the symmetry $B_{\text {if }}(-\mathbf{k}, \mathbf{e})=B_{\mathrm{fi}}(\mathbf{k}, \mathbf{e})$, which can easily be shown by partial integration, so that the induced absorption and emission probabilities are identical.

In dipole approximation, we may drop the exponential in (3.26), and use

$$
\int u_{\mathrm{f}}^{*} \mathbf{e} \cdot \nabla u_{\mathrm{i}} \mathrm{d} \mathbf{x}=\frac{m}{\hbar}\left(\omega_{\mathrm{f}}-\omega_{\mathrm{i}}\right) \int u_{\mathrm{f}}^{*} \mathbf{e} \cdot \mathbf{r} u_{\mathrm{i}} \mathrm{d} \mathbf{x} .
$$

The averaging over the transversal polarizations is performed by substituting

$$
\left|\int u_{\mathrm{f}}^{*} \mathbf{e} \cdot \mathbf{r} u_{\mathrm{i}} \mathrm{d} \mathbf{x}\right|^{2} \rightarrow \int\left|\int u_{\mathrm{f}}^{*} \mathbf{n} \cdot \mathbf{r}^{\prime} u_{\mathrm{i}} \mathrm{d} \mathbf{x}^{\prime}\right|^{2} \frac{\mathrm{d} \Omega}{4 \pi}=\frac{2}{3}\left|\int u_{\mathrm{f}}^{*} \mathbf{r}^{\prime} u_{\mathrm{i}} \mathrm{d} \mathbf{x}^{\prime}\right|^{2},
$$

cf. (3.14), and we so arrive at the $(\mathbf{e}, \mathbf{k} /|\mathbf{k}|)$-averaged, transition probability in dipole approximation,

$$
w_{\text {ind.em. }}^{\mathrm{d}}=w_{\text {ind.ab. }}^{\mathrm{d}} \sim B_{\text {if }}^{\mathrm{d}} \rho_{E}(\omega), \quad B_{\text {if }}^{\mathrm{d}}:=\frac{4 \pi^{2}}{3} \frac{1}{\hbar^{2}} \frac{q^{2}}{4 \pi}\left|\int u_{\mathrm{i}}^{*} \mathbf{r} u_{\mathrm{f}} \mathrm{d} \mathbf{x}\right|^{2} .
$$

Evidently, $B_{\text {if }}^{\mathrm{d}}=B_{\mathrm{fi}}^{\mathrm{d}}$. The Einstein coefficients (3.17), (3.20), (3.26) and (3.29) relate to each other via

$$
A_{\mathrm{if}}^{(\mathrm{d})}=\frac{\hbar k \omega^{2}}{\pi^{2} c^{2}} B_{\mathrm{if}}^{(\mathrm{d})}
$$


$k=\sqrt{\omega^{2} / c^{2}+\mu^{2}}, \mu=m_{\mathrm{t}} c / \hbar$, and they connect to the spectral energy density and the Boltzmann factor via the equilibrium condition

$$
B_{\text {if }}^{(\mathrm{d})} \rho_{E}(\omega)+A_{\text {if }}^{(\mathrm{d})}=B_{\text {if }}^{(\mathrm{d})} \rho_{E}(\omega) \exp (\beta \hbar \omega) .
$$

This is consistent with the transversal spectral energy density

$$
\rho_{E}(\omega)=\frac{\hbar}{\pi^{2} c^{2}} \frac{\omega^{2} \sqrt{\omega^{2} / c^{2}+\mu^{2}}}{\exp (\beta \hbar \omega)-1},
$$

which was derived by box quantization in Refs. [25], also see the beginning of Section 4. This density warrants scrutiny from various angles; a third derivation along the lines of Feinberg's quantization procedure [3] will be given elsewhere. His noninvariant vacuum can be easily defined in the comoving reference frame. This is another reason, apart from causality, advanced wavefields and the positivity of energy, to consider tachyons in an absolute cosmic space-time, propagating in a permeable ether.

The photonic transition rates and the Planck distribution are of course recovered by putting $\mu=0$ in $k=\sqrt{\omega^{2} / c^{2}+\mu^{2}}$. The emission and absorption rates for photon and tachyon radiation can be easily compared in the dipole approximation,

$$
\frac{w_{\text {sp.em. }}^{\mathrm{d}(\text { tach })}}{w_{\text {sp.em. }}^{\mathrm{d}(\mathrm{ph})}} \sim \frac{w_{\text {ind. }}^{\mathrm{d}(\text { tach })}}{w_{\text {ind. }}^{\mathrm{d}(\mathrm{ph})}} \sim \frac{q^{2}}{e^{2}} \frac{\sqrt{\omega^{2}+\mu^{2} c^{2}}}{\omega} ;
$$

otherwise the matrix elements enter,

$$
\frac{w_{\text {sp.em. }}^{(\text {tach })}}{w_{\text {sp.em. }}^{\text {(ph) }}} \sim \frac{w_{\text {ind. }}^{(\text {tach })}}{w_{\text {ind. }}^{(\text {ph })}} \sim \frac{q^{2}}{e^{2}} \frac{c k}{\omega} \frac{\left|\int u_{\mathrm{i}}^{*} \exp \left(-\mathrm{i} k \mathbf{n} \mathbf{x}^{\prime}\right) \mathbf{e} \cdot \nabla u_{\mathrm{f}} \mathrm{d} \mathbf{x}^{\prime}\right|^{2}}{\left|\int u_{\mathrm{i}}^{*} \exp \left(-\mathrm{i} \omega c^{-1} \mathbf{n} \mathbf{x}^{\prime}\right) \mathbf{e} \cdot \nabla u_{\mathrm{f}} \mathrm{d} \mathbf{x}^{\prime}\right|^{2}} .
$$

A quantitative discussion of (3.33) will be given in Section 6. For the dipole approximation to be valid, a large wavelength is required to justify the expansion of the exponentials. In Section 6 we will demonstrate, that the maximal wavelength a tachyon can attain is about $1 \AA$ (reduced Compton). This is not large compared to the support of the eigenfunctions, unless one considers heavy ions, but it is not small either, unless one considers Rydberg states, and so the averaging caused by the exponentials will be moderate in most cases. Hence, one may assume that (3.33) is a reasonable upper bound for (3.34), but a qualified comparison of (3.33) and (3.34) requires to specify the eigenfunctions in the integrals.

Finally we mention the changes needed for longitudinal transitions. In (3.20), the longitudinal $A$-coefficient is obtained by replacing e by $\mathbf{n}$. The spectral energy density for longitudinal tachyons is $\rho_{E} / 2$, with the transversal $\rho_{E}$ as in (3.32). The longitudinal $B$-coefficient is thus $2 B_{\text {if }}(\mathbf{k}, \mathbf{n})$, cf. (3.26). If we use the total spectral density $3 \rho_{E} / 2$ in the transition rates (3.24) and (3.25), the $B$-coefficient is of course $2 B_{\text {if }} / 3$ with the respective polarization.

As an example, we calculate the cross section in Born approximation for the tachyonic analog to the photoelectric effect. The transition probability per unit time from a bound state $u_{\mathrm{i}}$ in a hydrogen-like ion to a scattering state $u_{\mathrm{f}}$ is given by the semiclassical 
formula [35] (box-normalization is assumed, with box size $L$ )

$$
w \sim \frac{m k_{\mathrm{e}} L^{3}}{4 \pi^{2} \hbar^{3}}\left|\left\langle f\left|H_{\mathrm{int}}^{+}\right| \mathrm{i}\right\rangle\right|^{2} \mathrm{~d} \Omega,
$$

with $\left\langle f\left|H_{\text {int }}^{+}\right| \mathrm{i}\right\rangle$ as in (3.22). (In (3.34), both states $u_{\mathrm{i}, \mathrm{f}}$ are bound.) Electronic and tachyonic wave vectors and frequencies are denoted by $\mathbf{k}_{\mathrm{e}, \mathrm{t}}$ and $\omega_{\mathrm{e}, \mathrm{t}}$, respectively. ( $m$ is the electron mass, reduced mass corrections are neglected; the tachyon mass is denoted by $\left.m_{\mathrm{t}}.\right)$ The solid angle element $\mathrm{d} \Omega$ is centered at $\mathbf{k}_{\mathrm{e}}(\theta, \varphi)$. The final state in the continuous spectrum is approximated by a plane wave, $u_{\mathrm{f}} \sim L^{-3 / 2} \exp \left(i \mathbf{k}_{\mathrm{e}} \mathbf{x}\right)$. Hence,

$$
\left\langle f\left|H_{\text {int }}^{+}\right| \mathbf{i}\right\rangle \sim-\frac{q \hbar \mathbf{k}_{\mathrm{e}} \mathbf{e}}{m c L^{3 / 2}} \hat{a}^{\mathrm{T}} \int \exp (\mathrm{i} \mathbf{K} \mathbf{x}) u_{\mathrm{i}} \mathrm{d} \mathbf{x},
$$

with $\mathbf{K}:=\mathbf{k}_{\mathrm{t}}-\mathbf{k}_{\mathrm{e}}$, and $\mathbf{e}$ is the polarization unit vector of the transversal tachyon. The differential cross section, $\mathrm{d} \sigma=\sigma(\theta, \varphi) \mathrm{d} \Omega$, is obtained by dividing $w$ in (3.35) by the tachyonic flux, cf. (2.15),

$$
\begin{aligned}
& \frac{\left|\left\langle\mathbf{S}^{\mathrm{T}}\right\rangle\right|}{\hbar \omega_{\mathrm{t}}}=2 \frac{k_{\mathrm{t}}}{\hbar}\left|\hat{a}^{\mathrm{T}}\right|^{2}, \\
& \sigma(\theta, \varphi)=\frac{1}{2 \pi} \frac{1}{m c^{2}} \frac{q^{2}}{4 \pi} \frac{k_{\mathrm{e}}\left|\mathbf{k}_{\mathrm{e}} \mathbf{e}\right|^{2}}{k_{\mathrm{t}}}\left|\int \exp (\mathrm{i} \mathbf{K} \mathbf{x}) u_{\mathrm{i}} \mathrm{d} \mathbf{x}\right|^{2} .
\end{aligned}
$$

The ground state wave function of the unperturbed Coulomb problem (3.15) reads $u_{\mathrm{i}}^{(n=1, l=0)}:=\left(\pi a^{3}\right)^{-1 / 2} \mathrm{e}^{-r / a}$. Its range is determined by $a:=\hbar /(m c \alpha), \alpha:=Z e^{2} /(4 \pi \hbar c) \approx$ $Z / 137$, and we find

$$
\sigma(\theta, \varphi)=32 \frac{1}{m c^{2}} \frac{q^{2}}{4 \pi} \frac{k_{\mathrm{e}}}{k_{\mathrm{t}}}\left|\mathbf{k}_{\mathrm{e}} \mathbf{e}\right|^{2} \frac{a^{3}}{\left(a^{2} K^{2}+1\right)^{4}} .
$$

The Born approximation, i.e., the plane wave approximation of the scattering state, is safely valid for $k_{\mathrm{e}} a \gg 1$, which means a small wavelength compared to the range of $u_{\mathrm{i}}^{(n=1, l=0)}$. This is equivalent to $v / c \gg \alpha$ or $E_{1} \ll m v^{2} / 2$, with the ground state energy $E_{1}=m c^{2} \alpha^{2} / 2$. ( $v$ is the speed of the free electron.) On the other hand, $v / c \ll 1$ must hold, and energy conservation yields $\hbar^{2} k_{\mathrm{e}}^{2} /(2 m)=\hbar \omega_{\mathrm{t}}-E_{1}$. Hence, $m v^{2} / 2 \approx \hbar \omega_{\mathrm{t}}$, so that

$$
\frac{k_{\mathrm{t}}^{2}}{k_{\mathrm{e}}^{2}} \approx \frac{1}{2} \frac{\hbar \omega_{\mathrm{t}}}{m c^{2}}\left(1+\frac{\mu^{2} c^{2}}{\omega_{\mathrm{t}}^{2}}\right) \approx \frac{1}{4} \frac{v^{2}}{c^{2}}+\frac{m_{\mathrm{t}}^{2}}{m^{2}} \frac{c^{2}}{v^{2}},
$$

with the tachyon mass $\mu=m_{\mathrm{t}} c / \hbar$. We choose the polar axis in the direction $\mathbf{k}_{\mathrm{t}}$ of the incident tachyon, thus $K \approx k_{\mathrm{e}}\left(1-\left(k_{\mathrm{t}} / k_{\mathrm{e}}\right) \cos \theta\right)$, provided $k_{\mathrm{t}} / k_{\mathrm{e}} \ll 1$, cf. Section 6 . (In dipole approximation, $K \approx k_{\mathrm{e}}$.) Hence, $a K \gg 1$ in (3.39), and we find

$$
\sigma(\theta, \varphi) \approx 32 \frac{q^{2}}{4 \pi} \frac{1}{m c^{2}} \frac{1}{k_{\mathrm{t}} k_{\mathrm{e}}^{5} a^{5}} \frac{\sin ^{2} \theta \cos ^{2} \varphi}{\left(1-2\left(k_{\mathrm{t}} / k_{\mathrm{e}}\right) \cos \theta\right)^{4}},
$$

so that the total cross section and the maximum of $\sigma(\theta, \varphi)$ read, up to terms of $\mathrm{O}\left(k_{\mathrm{t}}^{2} / k_{\mathrm{e}}^{2}\right)$,

$$
\sigma \approx \frac{2^{7} \pi}{3} \frac{q^{2}}{4 \pi} \frac{1}{m c^{2}} \frac{1}{k_{\mathrm{t}}\left(k_{\mathrm{e}} a\right)^{5}}, \quad \theta_{\max } \approx \frac{\pi}{2}-4 \frac{k_{\mathrm{t}}}{k_{\mathrm{e}}} .
$$


The dipole approximation does not change $\sigma$ in the indicated order, but the $k$-term in $\theta_{\max }$ would be missing. The cross sections for the photoelectric effect are obtained by replacing $k_{\mathrm{t}}$ in the preceding formulas by $k_{\mathrm{ph}}=\omega_{\mathrm{ph}} / c$. The ratio of the tachyonic and photonic total cross sections and the relative shift of the maxima in the differential cross sections are given by

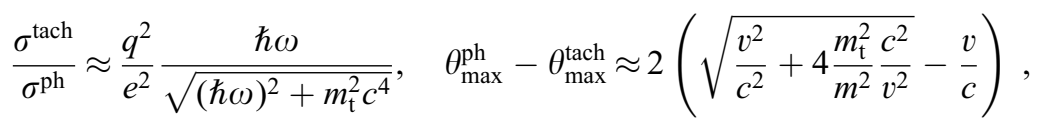

with $\omega=\omega_{\mathrm{ph}}=\omega_{\mathrm{t}}$. The forward shift of the maximum due to the retardation term $k_{\mathrm{t}} / k_{\mathrm{e}}$ is evidently more pronounced for tachyons. The terms under the roots can be of comparable magnitude in both formulas; quantitative estimates will be given in Section 6, cf. the discussion preceding (6.5).

The spherically symmetric, normalized bound states of hydrogen-like systems read as

$$
u_{\mathrm{i}}^{(n, l=0)}=\frac{1}{\sqrt{\pi} a_{n}^{3 / 2}} \frac{1}{n} L_{n-1}^{(1)}\left(2 r / a_{n}\right) \mathrm{e}^{-r / a_{n}}, \quad \frac{1}{n} L_{n-1}^{(1)}(x):=\frac{1}{x} \frac{\mathrm{e}^{x}}{n !} \frac{\mathrm{d}^{n-1}}{\mathrm{~d} x^{n-1}}\left(x^{n} \mathrm{e}^{-x}\right),
$$

$a_{n}:=a n$, and the corresponding energy levels are $E_{n}=E_{1} / n^{2}$. (The restriction to $l=0$ is not essential, but makes the integral in (3.38) handier.) The estimates following (3.39), including (3.40), stay valid with these replacements, that is, with the substitution $\alpha \rightarrow \alpha / n$. In particular, $k_{\mathrm{e}} a_{n} \gg 1$ is now the constraint for the applicability of the Born approximation. The differential cross section for the $n$th bound state reads, analogously to (3.39),

$$
\begin{aligned}
\sigma_{n}(\theta, \varphi)= & 8 \frac{1}{m c^{2}} \frac{q^{2}}{4 \pi} \frac{k_{\mathrm{e}}}{k_{\mathrm{t}}}\left|\mathbf{k}_{\mathrm{e}} \mathbf{e}\right|^{2} \frac{a_{n}}{K^{2}} \frac{1}{\left(K^{2} a_{n}^{2}+1\right)^{2(n+1)}} \operatorname{Im}^{2}\left(\left(1+\mathrm{i} a_{n} K\right)^{2 n}\right) \\
& \sim \frac{8}{n^{3}} \frac{1}{m c^{2}} \frac{q^{2}}{4 \pi} \frac{k_{\mathrm{e}}}{k_{\mathrm{t}}}\left|\mathbf{k}_{\mathrm{e}} \mathbf{e}\right|^{2} \frac{1}{a^{3} K^{6}} \sin ^{2}\left(\frac{2}{a K}\right),
\end{aligned}
$$

the asymptotics refers to large $n$, so that $a_{n} K \gg 1$. In Section 6 we will show, based on $k_{\mathrm{e}} a_{n} \gg 1$ and a tachyon mass of $2.1 \mathrm{keV} / c^{2}$, that $k_{\mathrm{t}} / k_{\mathrm{e}} \ll 1$ holds for the ground state. However, for highly excited states, $n \approx 10^{2-3}$, the opposite limit can be realized, $k_{\mathrm{t}} / k_{\mathrm{e}} \gg 1$.

The asymptotic limit of (3.45) for $a K \gg 1$ (and arbitrary $n$ ) is likewise (3.46), with the $\sin$ replaced by its leading order. In this case, if $k_{\mathrm{t}} / k_{\mathrm{e}} \ll 1$, we recover (3.41) apart from a scale factor $1 / n^{3}$, and (3.43) remains unchanged. If $k_{\mathrm{t}} / k_{\mathrm{e}} \gg 1$, we find instead

$$
\begin{gathered}
\sigma_{n}(\theta, \varphi) \approx \frac{32}{n^{3}} \frac{q^{2}}{4 \pi} \frac{1}{m c^{2}} \frac{k_{\mathrm{e}}^{3}}{k_{\mathrm{t}}^{9} a^{5}} \frac{\sin ^{2} \theta \cos ^{2} \varphi}{\left(1-2\left(k_{\mathrm{e}} / k_{\mathrm{t}}\right) \cos \theta\right)^{4}}, \\
\frac{\sigma_{n}^{\mathrm{tach}}}{\sigma_{n}^{\mathrm{ph}}} \approx \frac{q^{2}}{e^{2}}\left(\frac{k_{\mathrm{e}}}{k_{\mathrm{t}}}\right)^{8} \frac{k_{\mathrm{ph}}}{k_{\mathrm{t}}}, \quad \theta_{\max }^{\mathrm{ph}}-\theta_{\max }^{\mathrm{tach}} \approx 4 \frac{m}{m_{\mathrm{t}}} \frac{v}{c} .
\end{gathered}
$$

Here we have used, cf. (3.40), $k_{\mathrm{t}} / k_{\mathrm{e}} \approx m_{\mathrm{t}} c /(m v) \gg 1, k_{\mathrm{ph}} / k_{\mathrm{e}} \approx v /(2 c) \ll 1$, as well as $m_{\mathrm{t}} / m \ll 1$, cf. Section 6 . Clearly, the dipole approximation fails for $k_{\mathrm{t}} / k_{\mathrm{e}} \gg 1$. Finally, 
if $a K$ is moderate, the leading asymptotic order (3.46) can vanish due to zeros of the sin, and the opposite limit $a_{n} K \rightarrow 0$ is also admissible in (3.45), always with $k_{\mathrm{e}} a_{n} \gg 1$, but we will not pursue this further here. By the way, rainbow scattering in the static potential of this field theory is discussed in Ref. [25].

\section{The tachyon background: high- and low-temperature expansions of its internal energy}

The partition function of a tachyonic Bose gas [25] can be calculated via box quantization; we find for the transversal modes,

$$
\log Z=2 \log \sum_{\left(n_{p}\right)=0}^{\infty} \exp \left(-\beta \sum_{p} h v(p) n_{p}\right)=-2 \sum_{p} \log [1-\exp (-\beta h v(p))],
$$

$\beta^{-1}:=k T(\tau)=k T\left(\tau_{0}\right) / a(\tau)$. Here, $a(\tau)$ denotes the cosmic expansion factor in the Robertson-Walker geometry, and $\tau_{0}$ is the present epoch, $a\left(\tau_{0}\right)=1$. The chemical potential is zero, which follows from an equilibrium condition on the free energy, see the remark following Eq. (5.4). The index $p$ runs over a discrete set of values, $p=h L^{-1}\left(k_{1}, k_{2}, k_{3}\right), k_{i} \in Z, L$ the box size, and $\left(n_{p}\right)$ is a multi-index labeled by $p$. Euclidean box quantization with periodic boundary conditions also applies here, if the box size is much smaller than the curvature radius of the universe, see Refs. [27,37,38] for details and estimates with regard to the thermodynamic limit. The factor of two in (4.1) accounts for the two independent transversal states. This factor is absent in the partition function for the longitudinal modes, which is otherwise identical. Tachyon mass and frequencies conformally scale with the inverse of the expansion factor, and therefore this time dependence can be absorbed in the temperature variable as indicated. In the thermodynamic limit, the summation over the lattice points $p$ is replaced by an integral, and we arrive at

$$
\log Z=-\frac{2 V}{h^{3}} \int_{|p|>m_{\mathrm{t}} c} \mathrm{~d}^{3} p \log [1-\exp (-\beta h v(p))], \quad h v(p)=c \sqrt{|p|^{2}-\left(m_{\mathrm{t}} c\right)^{2}} .
$$

The thermodynamic limit does not depend on the curvature sign of the 3-space. If we put $m_{\mathrm{t}}=0$, Eq. (4.2) coincides with the partition function of the photon gas, of course. The transversal spectral energy density thus reads

$$
\rho(v) \mathrm{d} v=\frac{8 \pi V}{h^{3}} \frac{h v(p)|p|^{2} d|p|}{\exp [\beta h v(p)]-1},
$$

which is equivalent to (3.32) derived by means of detailed balancing and Einstein coefficients; also see the beginning of Section 6 for further discussion. The longitudinal 
and total spectral densities are $(1 / 2) \rho(v)$ and $(3 / 2) \rho(v)$, respectively, and the same factors apply to the internal and free energies and thus to all other thermodynamic variables calculated for the transversal modes in Section 5. The internal energy is obtained as

$$
\begin{aligned}
& U=\int_{0}^{\infty} \rho(v) \mathrm{d} v=8 \pi m_{\mathrm{t}}^{4} c^{5} h^{-3} V \hat{U}(\alpha), \\
& \hat{U}(\alpha):=\int_{0}^{\infty} \frac{x^{2} \sqrt{1+x^{2}} \mathrm{~d} x}{\exp (\alpha x)-1}, \quad \alpha:=\frac{m_{\mathrm{t}} c^{2}}{k T(\tau)}, \quad x:=\frac{h v}{m_{\mathrm{t}} c^{2}} .
\end{aligned}
$$

Next, we derive the high- and low-temperature expansions for the internal energy. The low-temperature expansion is easy to settle, given by the asymptotic series

$$
\hat{U}(\alpha) \sim \sum_{n=0}^{\infty}\left(\begin{array}{c}
1 / 2 \\
n
\end{array}\right) \int_{0}^{\infty} \frac{x^{2 n+2} \mathrm{~d} x}{\exp (\alpha x)-1}=\frac{1}{\alpha^{3}} \sum_{n=0}^{\infty}\left(\begin{array}{c}
1 / 2 \\
n
\end{array}\right) \Gamma(2 n+3) \zeta(2 n+3) \frac{1}{\alpha^{2 n}} .
$$

The justification of (4.5) follows from a mild modification of Watson's Lemma [39] on Laplace asymptotics. The first orders of the low-temperature expansion of the internal energy hence read

$$
U=16 \pi \zeta(3) \frac{m_{\mathrm{t}}^{4} c^{5} V}{h^{3}} \frac{1}{\alpha^{3}}\left[1+\frac{6 \zeta(5)}{\zeta(3)} \frac{1}{\alpha^{2}}-\frac{45 \zeta(7)}{\zeta(3)} \frac{1}{\alpha^{4}}+\mathrm{O}\left(\alpha^{-6}\right)\right] .
$$

To obtain the high-temperature expansion, we split the integral $\hat{U}(\alpha)$ into $\hat{U}(\alpha)=$ $\hat{U}_{0}(\alpha, \delta)+\hat{U}_{\infty}(\alpha, \delta)$, with

$$
\hat{U}_{0}(\alpha, \delta):=\frac{1}{\alpha^{4}} \int_{0}^{\delta} \frac{x^{2} \sqrt{x^{2}+\alpha^{2}} \mathrm{~d} x}{\mathrm{e}^{x}-1}, \quad \hat{U}_{\infty}(\alpha, \delta):=\frac{1}{\alpha^{4}} \int_{\delta}^{\infty} \frac{x^{3} \sqrt{1+\alpha^{2} / x^{2}} \mathrm{~d} x}{\mathrm{e}^{x}-1},
$$

and choose $\delta$ in the range $\alpha<\delta<2 \pi$. Expanding the root in $\hat{U}_{\infty}$, we arrive so at the absolutely convergent series

$$
\hat{U}_{\infty}(\alpha, \delta)=\frac{1}{\alpha^{4}} \sum_{n=0}^{\infty}\left(\begin{array}{c}
1 / 2 \\
n
\end{array}\right) \alpha^{2 n} \int_{\delta}^{\infty} \frac{x^{3-2 n} \mathrm{~d} x}{\mathrm{e}^{x}-1} .
$$

In $\hat{U}_{0}(\alpha, \delta)$, we substitute the generating series of the Bernoulli numbers,

$$
\frac{x}{\mathrm{e}^{x}-1}=\sum_{n=0}^{\infty} \frac{B_{n}}{\Gamma(n+1)} x^{n},
$$

which absolutely converges in $[0, \delta]$, and interchange summation and integration. We obtain in this way $\hat{U}_{0}(\alpha, \delta)=\hat{U}_{0}^{(1)}(\tilde{\alpha})+\hat{U}_{0}^{(2)}(\tilde{\alpha}, \delta), \tilde{\alpha}:=\alpha / \delta<1$,

$$
\begin{aligned}
\hat{U}_{0}^{(1)}(\tilde{\alpha}) & :=-\frac{1}{2 \tilde{\alpha}^{4}} \int_{0}^{1} x^{2} \sqrt{x^{2}+\tilde{\alpha}^{2}} \mathrm{~d} x \\
& =-\frac{1}{8} \frac{1}{\tilde{\alpha}^{4}} \sqrt{1+\tilde{\alpha}^{2}}\left(1+\tilde{\alpha}^{2} / 2\right)+\frac{1}{16} \log \left(\frac{1}{\tilde{\alpha}}\left(1+\sqrt{1+\tilde{\alpha}^{2}}\right)\right),
\end{aligned}
$$




$$
\begin{aligned}
& \log \left(1+\sqrt{1+\tilde{\alpha}^{2}}\right)=\log 2-\sum_{n=1}^{\infty} \frac{(-)^{n} \Gamma(2 n)}{2^{2 n} \Gamma^{2}(n+1)} \tilde{\alpha}^{2 n}, \\
& \hat{U}_{0}^{(2)}(\tilde{\alpha}, \delta):=\frac{1}{\delta \tilde{\alpha}^{4}} \sum_{k=0}^{\infty} \frac{B_{2 k} \delta^{2 k}}{\Gamma(2 k+1)} \int_{0}^{1} x^{2 k+1} \sqrt{x^{2}+\tilde{\alpha}^{2}} \mathrm{~d} x .
\end{aligned}
$$

A standard integral representation of the hypergeometric function, followed by a linear transformation of ${ }_{2} F_{1}$, gives

$$
\begin{aligned}
\int_{0}^{1} x^{2 k+1} \sqrt{x^{2}+\tilde{\alpha}^{2}} \mathrm{~d} x= & \frac{1}{2} \frac{\tilde{\alpha}}{k+1}{ }_{2} F_{1}\left(-1 / 2, k+1 ; k+2 ;-\tilde{\alpha}^{-2}\right) \\
= & \frac{1}{2 k+3}{ }_{2} F_{1}\left(-1 / 2,-k-3 / 2 ;-k-1 / 2 ;-\tilde{\alpha}^{2}\right) \\
& -\frac{\sqrt{\pi}}{4} \frac{\Gamma(k+1)(-)^{k}}{\Gamma(k+5 / 2)} \tilde{\alpha}^{2 k+3} .
\end{aligned}
$$

Since $\tilde{\alpha}<1$, we may use the absolutely convergent series representation

$$
\begin{gathered}
\frac{1}{2 k+3}{ }_{2} F_{1}\left(-1 / 2,-k-3 / 2 ;-k-1 / 2 ;-\tilde{\alpha}^{2}\right) \\
=-\frac{1}{2 \sqrt{\pi}} \sum_{n=0}^{\infty} \frac{\Gamma(n-1 / 2)(-)^{n} \tilde{\alpha}^{2 n}}{\Gamma(n+1)(2(k-n)+3)} .
\end{gathered}
$$

Inserting this into $\hat{U}_{0}^{(2)}$, and interchanging the summations, we arrive at

$$
\begin{aligned}
& \hat{U}_{0}^{(2)}(\tilde{\alpha}, \delta)=\hat{U}_{0}^{(2)(\text { even })}(\tilde{\alpha}, \delta)+\hat{U}_{0}^{(2)(\text { odd })}(\alpha), \\
& \hat{U}_{0}^{(2)(\text { even })}(\tilde{\alpha}, \delta):=-\frac{1}{2 \sqrt{\pi}} \frac{1}{\tilde{\alpha}^{4}} \sum_{n=0}^{\infty} \frac{\Gamma(n-1 / 2)}{\Gamma(n+1)} c_{n}(\delta)(-)^{n} \tilde{\alpha}^{2 n}, \\
& c_{n}(\delta):=\frac{1}{\delta} \sum_{k=0}^{\infty} \frac{B_{2 k} \delta^{2 k}}{\Gamma(2 k+1)(2(k-n)+3)}, \quad \delta<2 \pi, \\
& \hat{U}_{0}^{(2)(\text { odd })}(\alpha):=-\frac{\sqrt{\pi}}{4} \frac{1}{\alpha} \sum_{n=0}^{\infty} \frac{B_{2 n}}{\Gamma(2 n+1)} \frac{\Gamma(n+1)(-)^{n}}{\Gamma(n+5 / 2)} \alpha^{2 n} .
\end{aligned}
$$

We may write, using (4.9),

$$
c_{0}(\delta)=\frac{1}{\delta^{4}} \int_{0}^{\delta} \frac{x^{3} \mathrm{~d} x}{\mathrm{e}^{x}-1}+\frac{1}{8}, \quad c_{1}(\delta)=\frac{1}{\delta^{2}} \int_{0}^{\delta} \frac{x \mathrm{~d} x}{\mathrm{e}^{x}-1}+\frac{1}{4} .
$$

Collecting terms, we find

$$
\begin{aligned}
& \hat{U}(\alpha)=\hat{U}_{\infty}+\hat{U}_{0}^{(1)}+\hat{U}_{0}^{(2)(\text { even })}+\hat{U}_{0}^{(2)(\text { odd })}=: \hat{U}_{\text {sing }}(\alpha)+\hat{U}_{\text {reg }}(\alpha), \\
& \hat{U}_{\text {sing }}:=\frac{\pi^{4}}{15} \frac{1}{\alpha^{4}}+\frac{\pi^{2}}{12} \frac{1}{\alpha^{2}}-\frac{1}{3} \frac{1}{\alpha}-\frac{1}{16} \log \alpha, \quad \hat{U}_{\text {reg }}:=\sum_{n=0}^{\infty} a_{n} \alpha^{n} .
\end{aligned}
$$


The series $\hat{U}_{\text {reg }}$ converges for $\alpha<1$, and the result (4.15) can easily be extended to cover $\alpha<2 \pi$ [convergence radius of the series (4.9)], if we refrain from expanding certain elementary functions that appear in the above calculations. The coefficients of the odd powers in $\hat{U}_{\text {reg }}$ can be read off from $\hat{U}_{0}^{(2)(\text { odd })}$ in (4.13), and $a_{2 k}$ from $\hat{U}_{\infty}+$ $\hat{U}_{0}^{(1)}+\hat{U}_{0}^{(2)(\text { even })}$, cf. (4.8), (4.10) and (4.13). For example, we obtain

$$
a_{0}=\frac{1}{8}\left(-\frac{1}{8}+\frac{1}{2} \log (2 \delta)-c_{2}(\delta)-\int_{\delta}^{\infty} \frac{1}{\mathrm{e}^{x}-1} \frac{\mathrm{d} x}{x}\right) .
$$

Since $\hat{U}(\alpha)$ is independent of $\delta$, the same holds for the coefficients $a_{n}$. Hence, we may consider the limit $\delta \rightarrow 0$ in (4.16). Applying two times integration by parts, we find

$$
\begin{gathered}
\int_{\delta}^{\infty} \frac{x}{\mathrm{e}^{x}-1} \frac{1}{x^{2}} \mathrm{~d} x=\frac{1}{\delta}+\frac{1}{2} \log \delta-\frac{1}{2}+\gamma_{0}+\mathrm{O}(\delta), \\
\gamma_{0}:=-\int_{0}^{\infty} \frac{\mathrm{d}^{2}}{\mathrm{~d} x^{2}}\left(\frac{x}{\mathrm{e}^{x}-1}\right) \log x \mathrm{~d} x,
\end{gathered}
$$

and therefore

$$
a_{0}=\frac{1}{8}\left(\frac{3}{8}+\frac{1}{2} \log 2-\gamma_{0}\right) \text {. }
$$

$\delta$ can be eliminated from $a_{2 k}, k \geqslant 1$, in the same way. We apply $2(1+k)$ times partial integration to $\int_{\delta}^{\infty} x\left(\mathrm{e}^{x}-1\right)^{-1} x^{-2(1+k)} \mathrm{d} x$ in the series (4.8), and then perform the limit $\delta \rightarrow 0$, arriving so at a representation of the $a_{2 k}$ similar to (4.18).

The singular and the constant terms of the high-temperature expansion of $U$ give

$$
U=8 \pi \frac{m_{\mathrm{t}}^{4} c^{5} V}{h^{3}}\left[\frac{\pi^{4}}{15} \frac{1}{\alpha^{4}}+\frac{\pi^{2}}{12} \frac{1}{\alpha^{2}}-\frac{1}{3} \frac{1}{\alpha}-\frac{1}{16} \log \frac{\alpha}{2}+\frac{3}{64}-\frac{1}{8} \gamma_{0}+\mathrm{O}(\alpha)\right] .
$$

The internal energy will be discussed in Section 5 , in a more complete thermodynamic setting.

\section{Equilibrium thermodynamics of a free tachyon gas}

In this section, we evaluate the partition function of the tachyon gas, and all that goes with it. We already derived, cf. Eq. (4.2),

$$
\log Z=-\frac{8 \pi V}{h^{3}} \int_{m_{\mathrm{t}} c}^{\infty}|p|^{2} \mathrm{~d}|p| \log \left[1-\exp \left(-\alpha \sqrt{|p|^{2} /\left(m_{\mathrm{t}} c\right)^{2}-1}\right)\right],
$$

with $\alpha=m_{\mathrm{t}} c^{2} /(k T)$. The free energy of transversal tachyons thus reads

$$
\begin{aligned}
& F=-\frac{m_{\mathrm{t}} c^{2}}{\alpha} \log Z=-\frac{8 \pi}{3} \frac{m_{\mathrm{t}}^{4} c^{5}}{h^{3}} V \hat{F}(\alpha), \\
& \hat{F}(\alpha):=-\frac{3}{\alpha} \int_{0}^{\infty} x \sqrt{x^{2}+1} \log \left(1-\mathrm{e}^{-\alpha x}\right) \mathrm{d} x=\int_{0}^{\infty} \frac{\left(x^{2}+1\right)^{3 / 2}-1}{\mathrm{e}^{\alpha x}-1} \mathrm{~d} x,
\end{aligned}
$$


where we used partial integration and the elementary antiderivative of $\left(e^{\alpha x}-1\right)^{-1}$. Internal energy and entropy are related to $\hat{F}(\alpha)$ via

$$
\begin{aligned}
& U=\frac{\partial(\alpha F)}{\partial \alpha}=8 \pi \frac{m_{\mathrm{t}}^{4} c^{5}}{h^{3}} V \hat{U}(\alpha), \quad \hat{U}(\alpha)=-\frac{1}{3} \frac{\partial(\alpha \hat{F}(\alpha))}{\partial \alpha}, \\
& S=-\frac{\partial F}{\partial T}=-\frac{8 \pi}{3} \frac{k m_{\mathrm{t}}^{3} c^{3}}{h^{3}} V \alpha^{2} \frac{\partial \hat{F}(\alpha)}{\partial \alpha} .
\end{aligned}
$$

In fact, $F$ can almost be obtained using term by term integration of the series expansions derived for $\hat{U}(\alpha)$ in Section 4, apart from the $\alpha^{-1}$-term, determined by the integration constant. [The relevant quantities are of course $u=U / V$ and $s=S / V$, but we write in the following the volume factor and capital letters to avoid notational confusion.]

Remark. We have $U=F+T S$, and, since $\partial F(T, V, N) / \partial N=\mu=0$, the pressure reads $P=-F / V$. The condition $\partial F / \partial N=0$ is necessary to impose. Tachyons, like photons, are not interacting with each other. Thus, for equilibrium to be reached, we must assume interaction with subluminal matter, absorption and emission processes. Therefore $N$ cannot be kept constant, and $\partial F / \partial N=0$ is a necessary extremal condition for equilibrium. Accordingly, we have to put $\mu=0$ in the partition function. (The chemical potential should not be confused with the abbreviation for $m_{\mathrm{t}} c / \hbar$, used in Sections 2 and 3.) The tachyon number density $N / V$ is calculated below as a function of temperature, cf. Eq. (5.23).

As the chemical potential vanishes, the enthalpy just reads $H=T S$. We find for the heat capacities

$$
\begin{aligned}
& c_{V}=T \frac{\partial S}{\partial T}=\frac{\partial U}{\partial T}=-\frac{k}{m_{\mathrm{t}} c^{2}} \alpha^{2} \frac{\partial U}{\partial \alpha}=-\alpha \frac{\partial S}{\partial \alpha}, \\
& c_{P}=\frac{\partial H}{\partial T}=c_{V}+S=-\alpha^{2} \frac{\partial\left(\alpha^{-1} S\right)}{\partial \alpha}, \quad \gamma=\frac{c_{P}}{c_{V}}=1-\left(\frac{\alpha}{S} \frac{\partial S}{\partial \alpha}\right)^{-1} .
\end{aligned}
$$

The low- and high-temperature expansions of $F$ and $N$ can be derived quite similarly to those of $U$, and so we will indicate just the main steps without further comments. The low-temperature expansion of $\hat{F}$ reads as

$$
\hat{F}(\alpha) \sim \sum_{n=1}^{\infty}\left(\begin{array}{c}
3 / 2 \\
n
\end{array}\right) \int_{0}^{\infty} \frac{x^{2 n} \mathrm{~d} x}{\mathrm{e}^{\alpha x}-1}=\sum_{n=0}^{\infty}\left(\begin{array}{c}
3 / 2 \\
n+1
\end{array}\right) \Gamma(2 n+3) \zeta(2 n+3) \frac{1}{\alpha^{2 n+3}},
$$

from which we readily obtain

$$
\begin{aligned}
& S=24 \pi \zeta(3) \frac{k m_{\mathrm{t}}^{3} c^{3} V}{h^{3}} \frac{1}{\alpha^{2}}\left[1+\frac{5 \zeta(5)}{\zeta(3)} \frac{1}{\alpha^{2}}-\frac{35 \zeta(7)}{\zeta(3)} \frac{1}{\alpha^{4}}+\mathrm{O}\left(\alpha^{-6}\right)\right], \\
& P=-\frac{F}{V}=8 \pi \zeta(3) \frac{m_{\mathrm{t}}^{4} c^{5}}{h^{3}} \frac{1}{\alpha^{3}}\left[1+\frac{3 \zeta(5)}{\zeta(3)} \frac{1}{\alpha^{2}}-\frac{15 \zeta(7)}{\zeta(3)} \frac{1}{\alpha^{4}}+\mathrm{O}\left(\alpha^{-6}\right)\right],
\end{aligned}
$$




$$
\begin{aligned}
& c_{V}=48 \pi \zeta(3) \frac{k m_{\mathrm{t}}^{3} c^{3} V}{h^{3}} \frac{1}{\alpha^{2}}\left[1+\frac{10 \zeta(5)}{\zeta(3)} \frac{1}{\alpha^{2}}-\frac{105 \zeta(7)}{\zeta(3)} \frac{1}{\alpha^{4}}+\mathrm{O}\left(\alpha^{-6}\right)\right], \\
& c_{P}=72 \pi \zeta(3) \frac{k m_{\mathrm{t}}^{3} c^{3} V}{h^{3}} \frac{1}{\alpha^{2}}\left[1+\frac{25 \zeta(5)}{3 \zeta(3)} \frac{1}{\alpha^{2}}-\frac{245 \zeta(7)}{3 \zeta(3)} \frac{1}{\alpha^{4}}+\mathrm{O}\left(\alpha^{-6}\right)\right], \\
& \gamma=\frac{3}{2}\left[1-\frac{5 \zeta(5)}{3 \zeta(3)} \frac{1}{\alpha^{2}}+\left(\frac{70 \zeta(7)}{3 \zeta(3)}+\frac{50 \zeta^{2}(5)}{3 \zeta^{2}(3)}\right) \frac{1}{\alpha^{4}}+\mathrm{O}\left(\alpha^{-6}\right)\right] .
\end{aligned}
$$

In the high-temperature limit, $\alpha \rightarrow 0, \hat{F}$ is calculated as follows: $\hat{F}=\hat{F}_{0}+\hat{F}_{\infty}$,

$$
\begin{aligned}
\hat{F}_{0} & :=\frac{1}{\alpha^{4}} \int_{0}^{\delta} \frac{\left(x^{2}+\alpha^{2}\right)^{3 / 2}-\alpha^{3}}{\mathrm{e}^{x}-1} \mathrm{~d} x, \quad \hat{F}_{\infty}:=\frac{1}{\alpha^{4}} \int_{\delta}^{\infty} \frac{x^{3}\left(1+\alpha^{2} / x^{2}\right)^{3 / 2}-\alpha^{3}}{\mathrm{e}^{x}-1} \mathrm{~d} x \\
\hat{F}_{\infty} & =\frac{1}{\alpha}\left(-\delta+\log \left(\mathrm{e}^{\delta}-1\right)\right)+\frac{1}{\alpha^{4}} \sum_{n=0}^{\infty}\left(\begin{array}{c}
3 / 2 \\
n
\end{array}\right) \alpha^{2 n} \int_{\delta}^{\infty} \frac{x^{3-2 n} \mathrm{~d} x}{\mathrm{e}^{x}-1}, \\
\hat{F}_{0} & =\hat{F}_{0}^{(1)}+\hat{F}_{0}^{(2)}, \quad \hat{F}_{0}^{(1)}:=-\frac{1}{2 \tilde{\alpha}^{4}} \int_{0}^{1}\left[\left(x^{2}+\tilde{\alpha}^{2}\right)^{3 / 2}-\tilde{\alpha}^{3}\right] \mathrm{d} x \\
& =-\frac{1}{8} \frac{1}{\tilde{\alpha}^{4}} \sqrt{1+\tilde{\alpha}^{2}}-\frac{5}{16} \frac{1}{\tilde{\alpha}^{2}} \sqrt{1+\tilde{\alpha}^{2}}+\frac{1}{2} \frac{1}{\tilde{\alpha}}-\frac{3}{16} \log \left(\frac{1}{\tilde{\alpha}}\left(1+\sqrt{1+\tilde{\alpha}^{2}}\right)\right), \\
\hat{F}_{0}^{(2)} & :=\frac{1}{\delta \tilde{\alpha}^{4}} \sum_{k=0}^{\infty} \frac{B_{2 k} \delta^{2 k}}{\Gamma(2 k+1)} \int_{0}^{1} x^{2 k-1}\left[\left(x^{2}+\tilde{\alpha}^{2}\right)^{3 / 2}-\tilde{\alpha}^{3}\right] \mathrm{d} x
\end{aligned}
$$

with $\tilde{\alpha}:=\alpha / \delta<1$. We split the series $\hat{F}_{0}^{(2)}$ further, using for $k \geqslant 1$ the series expansion

$$
\begin{aligned}
\int_{0}^{1} x^{2 k-1}\left(x^{2}+\tilde{\alpha}^{2}\right)^{3 / 2} \mathrm{~d} x= & \frac{\tilde{\alpha}^{3}}{2 k}{ }_{2} F_{1}\left(-3 / 2, k ; k+1 ;-\tilde{\alpha}^{-2}\right) \\
= & \frac{3}{4 \sqrt{\pi}} \sum_{n=0}^{\infty} \frac{\Gamma(n-3 / 2)(-)^{n} \tilde{\alpha}^{2 n}}{\Gamma(n+1)(2(k-n)+3)} \\
& +\frac{3}{8 \sqrt{\pi}} \Gamma(k) \Gamma(-k-3 / 2) \tilde{\alpha}^{2 k+3} .
\end{aligned}
$$

By interchanging summations, we arrive at

$$
\begin{aligned}
\hat{F}_{0}^{(2)}=\hat{F}_{0}^{(2)(\mathrm{a})}+\hat{F}_{0}^{(2)(\mathrm{b})}+\hat{F}_{0}^{(2)(\text { even })}+\hat{F}_{0}^{(2)(\text { odd })} \\
\hat{F}_{0}^{(2)(\mathrm{a})}:=\frac{1}{\delta \tilde{\alpha}^{4}} \int_{0}^{1}\left[\left(x^{2}+\tilde{\alpha}^{2}\right)^{3 / 2}-\tilde{\alpha}^{3}\right] \frac{\mathrm{d} x}{x}=\frac{1}{\delta}\left[\frac{1}{3} \frac{1}{\tilde{\alpha}^{4}}\left(1+\tilde{\alpha}^{2}\right)^{3 / 2}\right. \\
\left.+\frac{1}{\tilde{\alpha}^{2}} \sqrt{1+\tilde{\alpha}^{2}}+\frac{1}{\tilde{\alpha}}\left(-\frac{4}{3}+\log (2 \tilde{\alpha})-\log \left(\tilde{\alpha}+\sqrt{1+\tilde{\alpha}^{2}}\right)\right)\right],
\end{aligned}
$$




$$
\begin{aligned}
& \log \left(\tilde{\alpha}+\sqrt{1+\tilde{\alpha}^{2}}\right)=\sum_{n=0}^{\infty} \frac{(-)^{n} \Gamma(2 n+1)}{2^{2 n} \Gamma^{2}(n+1)(2 n+1)} \tilde{\alpha}^{2 n+1}, \\
& \hat{F}_{0}^{(2)(\mathrm{b})}:=-\frac{1}{\alpha} \sum_{n=1}^{\infty} \frac{B_{2 n} \delta^{2 n}}{\Gamma(2 n+1) 2 n}=\frac{1}{\alpha}\left(\frac{1}{2} \delta-\log \left(\mathrm{e}^{\delta}-1\right)+\log \delta\right), \\
& \hat{F}_{0}^{(2)(\text { even })}:=\frac{3}{4 \sqrt{\pi}} \frac{1}{\alpha^{4}} \sum_{n=0}^{\infty} \tilde{c}_{n}(\delta) \frac{\Gamma(n-3 / 2)}{\Gamma(n+1)}(-)^{n} \tilde{\alpha}^{2 n}, \quad \tilde{c}_{n}(\delta):=c_{n}(\delta)+\frac{1}{2 n-3} \frac{1}{\delta}, \\
& \hat{F}_{0}^{(2)(\text { odd })}:=\frac{3}{8 \sqrt{\pi}} \frac{1}{\alpha} \sum_{n=1}^{\infty} \frac{B_{2 n}}{\Gamma(2 n+1)} \Gamma(n) \Gamma(-n-3 / 2) \alpha^{2 n} .
\end{aligned}
$$

The coefficients $\tilde{c}_{0}(\delta)$ and $\tilde{c}_{1}(\delta)$ can be immediately read off from (4.14), and by combining (4.16) and (4.18) we obtain

$$
\tilde{c}_{2}(\delta)=\frac{1}{\delta}-\frac{1}{2}+\gamma_{0}+\frac{1}{2} \log \delta-\int_{\delta}^{\infty} \frac{1}{\mathrm{e}^{x}-1} \frac{\mathrm{d} x}{x} .
$$

Collecting terms, we find

$$
\begin{aligned}
& \hat{F}=\hat{F}_{\infty}+\hat{F}_{0}^{(1)}+\hat{F}_{0}^{(2)(\mathrm{a})}+\hat{F}_{0}^{(2)(\mathrm{b})}+\hat{F}_{0}^{(2)(\mathrm{even})}+\hat{F}_{0}^{(2)(\text { odd })}=\hat{F}_{\text {sing }}(\alpha)+\hat{F}_{\text {reg }}(\alpha), \\
& \hat{F}_{\text {sing }}:=\frac{\pi^{4}}{15} \frac{1}{\alpha^{4}}+\frac{\pi^{2}}{4} \frac{1}{\alpha^{2}}+\frac{1}{\alpha}\left(-\frac{4}{3}+\log (2 \alpha)\right)+\frac{3}{16} \log \alpha \\
& \hat{F}_{\text {reg }}:=\sum_{n=0}^{\infty} b_{n} \alpha^{n}, \quad b_{0}=-\frac{21}{64}-\frac{3}{16} \log 2+\frac{3}{8} \gamma_{0} .
\end{aligned}
$$

The series $\hat{F}_{\text {reg }}$ converges for $\alpha<1$, see after (4.15). The high-temperature expansions of entropy, pressure, and specific heat hence read

$$
\begin{aligned}
S= & 8 \pi \frac{m_{\mathrm{t}}^{3} c^{3} k V}{h^{3}}\left[\frac{4 \pi^{4}}{45} \frac{1}{\alpha^{3}}+\frac{\pi^{2}}{6} \frac{1}{\alpha}+\frac{1}{3} \log (2 \alpha)-\frac{7}{9}-\frac{\alpha}{16}+\mathrm{O}\left(\alpha^{2}\right)\right], \\
P= & 8 \pi \frac{m_{\mathrm{t}}^{4} c^{5}}{h^{3}}\left[\frac{\pi^{4}}{45} \frac{1}{\alpha^{4}}+\frac{\pi^{2}}{12} \frac{1}{\alpha^{2}}+\frac{1}{\alpha}\left(-\frac{4}{9}+\frac{1}{3} \log (2 \alpha)\right)\right. \\
& \left.+\frac{1}{16} \log \frac{\alpha}{2}-\frac{7}{64}+\frac{1}{8} \gamma_{0}+\mathrm{O}(\alpha)\right], \\
c_{V}= & 8 \pi \frac{m_{\mathrm{t}}^{3} c^{3} k V}{h^{3}}\left[\frac{4 \pi^{4}}{15} \frac{1}{\alpha^{3}}+\frac{\pi^{2}}{6} \frac{1}{\alpha}-\frac{1}{3}+\frac{\alpha}{16}+\mathrm{O}\left(\alpha^{2}\right)\right], \\
c_{P}= & 8 \pi \frac{m_{\mathrm{t}}^{3} c^{3} k V}{h^{3}}\left[\frac{16 \pi^{4}}{45} \frac{1}{\alpha^{3}}+\frac{\pi^{2}}{3} \frac{1}{\alpha}+\frac{1}{3} \log (2 \alpha)-\frac{10}{9}+\mathrm{O}\left(\alpha^{2}\right)\right],
\end{aligned}
$$




$$
\gamma=\frac{4}{3}\left[1+\frac{5}{16 \pi^{2}} \alpha^{2}-\frac{15}{8 \pi^{4}} \alpha^{3}\left(1-\frac{1}{2} \log (2 \alpha)\right)-\frac{55}{128 \pi^{4}} \alpha^{4}+\mathrm{O}\left(\alpha^{5}\right)\right] .
$$

The particle number, which is, as pointed out in the Remark following (5.4), not an independent variable, is obtained as, cf. Eqs. (4.3) and (4.4),

$$
\begin{aligned}
& N=\frac{8 \pi V}{h^{3}} \int_{m_{\mathrm{t}} c}^{\infty} \frac{|p|^{2} \mathrm{~d}|p|}{\exp \left[\alpha \sqrt{|p|^{2} /\left(m_{\mathrm{t}} c\right)^{2}-1}\right]-1}=\int_{0}^{\infty} n(v) \mathrm{d} v=8 \pi \frac{m_{\mathrm{t}}^{3} c^{3}}{h^{3}} V \hat{N}(\alpha), \\
& \hat{N}(\alpha):=\int_{0}^{\infty} \frac{\sqrt{x^{2}+1}}{\mathrm{e}^{\alpha x}-1} x \mathrm{~d} x, \quad x=\frac{h v}{m_{\mathrm{t}} c^{2}} .
\end{aligned}
$$

For $\alpha \rightarrow \infty$, we immediately find

$$
\begin{aligned}
& \hat{N} \sim \sum_{n=0}^{\infty}\left(\begin{array}{c}
1 / 2 \\
n
\end{array}\right) \int_{0}^{\infty} \frac{x^{2 n+1} \mathrm{~d} x}{\mathrm{e}^{\alpha x}-1}=\frac{\pi^{2}}{\alpha^{2}} \sum_{n=0}^{\infty}\left(\begin{array}{c}
1 / 2 \\
n
\end{array}\right) \frac{(2 \pi)^{2 n} B_{2(n+1)}}{n+1} \frac{(-)^{n}}{\alpha^{2 n}}, \\
& N=\frac{4}{3} \pi^{3} \frac{m_{\mathrm{t}}^{3} c^{3} V}{h^{3}} \frac{1}{\alpha^{2}}\left[1+\frac{\pi^{2}}{5} \frac{1}{\alpha^{2}}-\frac{2 \pi^{4}}{21} \frac{1}{\alpha^{4}}+\mathrm{O}\left(\alpha^{-6}\right)\right] .
\end{aligned}
$$

Combining this with (4.6), we obtain the energy per tachyon as a function of temperature,

$$
\begin{aligned}
\frac{U}{N}= & \frac{12 \zeta(3) m_{\mathrm{t}} c^{2}}{\pi^{2}} \frac{1}{\alpha}\left[1+\left(\frac{6 \zeta(5)}{\zeta(3)}-\frac{\pi^{2}}{5}\right) \frac{1}{\alpha^{2}}\right. \\
& \left.+\left(\frac{71 \pi^{4}}{525}-\frac{45 \zeta(7)}{\zeta(3)}-\frac{6 \pi^{2} \zeta(5)}{5 \zeta(3)}\right) \frac{1}{\alpha^{4}}+\mathrm{O}\left(\alpha^{-6}\right)\right],
\end{aligned}
$$

and the entropy per tachyon reads, cf. (5.8) and (5.25),

$$
\begin{aligned}
\frac{S}{N}= & \frac{18 \zeta(3) k}{\pi^{2}}\left[1+\left(\frac{5 \zeta(5)}{\zeta(3)}-\frac{\pi^{2}}{5}\right) \frac{1}{\alpha^{2}}\right. \\
& \left.+\left(\frac{71 \pi^{4}}{525}-\frac{35 \zeta(7)}{\zeta(3)}-\frac{\pi^{2} \zeta(5)}{\zeta(3)}\right) \frac{1}{\alpha^{4}}+\mathrm{O}\left(\alpha^{-6}\right)\right] .
\end{aligned}
$$

In the high-temperature regime, $\alpha \rightarrow 0$, we write $\hat{N}(\alpha)$ in $(4.23)$ as $\hat{N}(\alpha)=\hat{N}_{0}+\hat{N}_{\infty}$,

$$
\begin{aligned}
& \hat{N}_{0}:=\frac{1}{\alpha^{3}} \int_{0}^{\delta} \frac{x \sqrt{x^{2}+\alpha^{2}} \mathrm{~d} x}{\mathrm{e}^{x}-1}, \quad \hat{N}_{\infty}:=\frac{1}{\alpha^{3}} \int_{\delta}^{\infty} \frac{x^{2} \sqrt{1+\alpha^{2} / x^{2}} \mathrm{~d} x}{\mathrm{e}^{x}-1}, \\
& \hat{N}_{\infty}=\frac{1}{\alpha^{3}} \sum_{n=0}^{\infty}\left(\begin{array}{c}
1 / 2 \\
n
\end{array}\right) \alpha^{2 n} \int_{\delta}^{\infty} \frac{x^{2-2 n} \mathrm{~d} x}{\mathrm{e}^{x}-1}, \quad \hat{N}_{0}=\hat{N}_{0}^{(1)}+\hat{N}_{0}^{(2)}, \\
& \hat{N}_{0}^{(1)}:=-\frac{1}{2 \tilde{\alpha}^{3}} \int_{0}^{1} x \sqrt{x^{2}+\tilde{\alpha}^{2}} \mathrm{~d} x=-\frac{1}{6} \frac{1}{\tilde{\alpha}^{3}}\left(1+\tilde{\alpha}^{2}\right)^{3 / 2}+\frac{1}{6}, \\
& \hat{N}_{0}^{(2)}:=\frac{1}{\delta \tilde{\alpha}^{3}} \sum_{k=0}^{\infty} \frac{B_{2 k} \delta^{2 k}}{\Gamma(2 k+1)} \int_{0}^{1} x^{2 k} \sqrt{x^{2}+\tilde{\alpha}^{2}} \mathrm{~d} x .
\end{aligned}
$$


To obtain power series for the integrals in $\hat{N}_{0}^{(2)}$, we note

$$
\begin{aligned}
\int_{0}^{1} x^{2(k+\varepsilon)} \sqrt{x^{2}+\tilde{\alpha}^{2}} \mathrm{~d} x= & \frac{\tilde{\alpha}}{2(k+\varepsilon)+1}{ }_{2} F_{1}\left(-\frac{1}{2}, k+\varepsilon+\frac{1}{2} ; k+\varepsilon+\frac{3}{2} ;-\tilde{\alpha}^{-2}\right) \\
= & \frac{1}{2(k+\varepsilon+1)}{ }_{2} F_{1}\left(-\frac{1}{2},-k-\varepsilon-1 ;-k-\varepsilon ;-\tilde{\alpha}^{2}\right) \\
& +\frac{\sqrt{\pi}}{4} \frac{\Gamma(k+\varepsilon+1 / 2)}{\Gamma(k+\varepsilon+2) \sin (\pi(k+\varepsilon+1))} \tilde{\alpha}^{2(k+\varepsilon)+2} .
\end{aligned}
$$

By expanding the right-hand side of (5.29) in powers of $\varepsilon$, and then performing the limit $\varepsilon \rightarrow 0$, we arrive at

$$
\begin{gathered}
\int_{0}^{1} x^{2 k} \sqrt{x^{2}+\tilde{\alpha}^{2}} \mathrm{~d} x=-\frac{1}{4 \sqrt{\pi}} \sum_{\substack{n=0 \\
n \neq k+1}}^{\infty} \frac{\Gamma(n-1 / 2)(-)^{n} \tilde{\alpha}^{2 n}}{\Gamma(n+1)(k-n+1)} \\
+\frac{(-)^{k}}{4 \sqrt{\pi}} \frac{\Gamma(k+1 / 2) \tilde{\alpha}^{2(k+1)}}{\Gamma(k+2)}(\psi(k+2)-\psi(k+1 / 2)-2 \log \tilde{\alpha}) .
\end{gathered}
$$

[ $\psi$, the logarithmic derivative of $\Gamma$, is elementary for positive (half-)integers.] Inserting this into $\hat{N}_{0}^{(2)}$, and interchanging summations, we obtain $\hat{N}_{0}^{(2)}=\hat{N}_{0}^{(2)(a)}+\hat{N}_{0}^{(2)(b)}$,

$$
\begin{aligned}
\hat{N}_{0}^{(2)(\mathrm{a})}:= & -\frac{1}{2 \sqrt{\pi}} \frac{1}{\tilde{\alpha}^{3}} \sum_{n=0}^{\infty} \frac{\Gamma(n-1 / 2)}{\Gamma(n+1)} \hat{c}_{n}(\delta)(-)^{n} \tilde{\alpha}^{2 n}, \\
\hat{c}_{n}(\delta):= & \frac{1}{2 \delta} \sum_{\substack{k=0 \\
k \neq n-1}}^{\infty} \frac{B_{2 k} \delta^{2 k}}{\Gamma(2 k+1)(k-n+1)}, \\
\hat{N}_{0}^{(2)(\mathrm{b})}:= & \frac{1}{4 \sqrt{\pi}} \frac{1}{\alpha} \sum_{n=0}^{\infty} \frac{B_{2 n}}{\Gamma(2 n+1)} \frac{\Gamma(n+1 / 2)}{\Gamma(n+2)} \\
& \times(\psi(n+2)-\psi(n+1 / 2)-2 \log \tilde{\alpha})(-)^{n} \alpha^{2 n} .
\end{aligned}
$$

Since

$$
\hat{c}_{0}(\delta)=\frac{1}{\delta^{3}} \int_{0}^{\delta} \frac{x^{2} \mathrm{~d} x}{\mathrm{e}^{x}-1}+\frac{1}{6}, \quad \hat{c}_{1}(\delta)=\frac{1}{\delta} \log \left(\mathrm{e}^{\delta}-1\right)-\frac{1}{\delta} \log \delta-\frac{1}{2},
$$

we find, via (5.23) and $\hat{N}=\hat{N}_{\infty}+\hat{N}_{0}^{(1)}+\hat{N}_{0}^{(2)(a)}+\hat{N}_{0}^{(2)(b)}$, the particle density as

$$
\frac{N}{V}=8 \pi \frac{m_{\mathrm{t}}^{3} c^{3}}{h^{3}}\left[\frac{2 \zeta(3)}{\alpha^{3}}+\frac{1}{\alpha}\left(\frac{1}{4}-\frac{1}{2} \log \frac{\alpha}{2}\right)+\frac{1}{6}+\mathrm{O}(\alpha \log \alpha)\right] .
$$


The high-temperature expansion of the energy per particle is obtained from (4.19) and (5.33),

$$
\begin{aligned}
\frac{U}{N}= & \frac{\pi^{4} m_{\mathrm{t}} c^{2}}{30 \zeta(3)} \frac{1}{\alpha}\left[1+\left(\frac{5}{4 \pi^{2}}-\frac{1}{8 \zeta(3)}+\frac{1}{4 \zeta(3)} \log \frac{\alpha}{2}\right) \alpha^{2}\right. \\
& \left.-\left(\frac{5}{\pi^{4}}+\frac{1}{12 \zeta(3)}\right) \alpha^{3}+\mathrm{O}\left(\alpha^{4} \log ^{2} \alpha\right)\right]
\end{aligned}
$$

and the entropy per particle reads in this limit, cf. Eq. (5.18),

$$
\begin{aligned}
\frac{S}{N}= & \frac{2 \pi^{4} k}{45 \zeta(3)}\left[1+\left(\frac{15}{8 \pi^{2}}-\frac{1}{8 \zeta(3)}+\frac{1}{4 \zeta(3)} \log \frac{\alpha}{2}\right) \alpha^{2}\right. \\
& \left.-\left(\frac{35}{4 \pi^{4}}+\frac{1}{12 \zeta(3)}-\frac{15}{4 \pi^{4}} \log (2 \alpha)\right) \alpha^{3}+\mathrm{O}\left(\alpha^{4} \log ^{2} \alpha\right)\right] .
\end{aligned}
$$

The mass drops out in the leading order of the expansions (4.19), (5.18)-(5.22), and (5.33)-(5.35), and hence the high-temperature limit coincides with electromagnetic black-body radiation. It is only in the low-temperature regime, that the tachyon mass changes the temperature scaling in leading order, cf. (4.6), (5.8)-(5.12), and (5.25)(5.27),

$$
\begin{aligned}
& U \sim 16 \pi \zeta(3) \frac{m_{\mathrm{t}} k^{3}}{c h^{3}} V T^{3}, \quad N \sim \frac{4}{3} \pi^{3} \frac{m_{\mathrm{t}} k^{2}}{c h^{3}} V T^{2}, \\
& P V \sim \frac{1}{2} U \sim \frac{6 \zeta(3)}{\pi^{2}} N k T, \quad S \sim \frac{1}{2} c_{V} \sim \frac{3}{2} \frac{U}{T} \sim \frac{18 \zeta(3)}{\pi^{2}} N k, \quad \gamma \sim \frac{3}{2},
\end{aligned}
$$

with $\zeta(3) \approx 1.202$. The entropy and the thermal equation of state are also in this limit independent of the tachyon mass, but not so the caloric equation. These relations bear little resemblance to those of a relativistic gas of subluminal massive particles, regardless of the statistics used and the limits considered [38], as $m_{\mathrm{t}}$ is not a rest mass and the chemical potential is zero. In the next section, we will turn to quantitative estimates, and discuss the prospects to observe the cosmic tachyon background.

\section{Conclusion}

The spectral energy density of the transversal tachyon radiation reads, cf. Eqs. (3.32) and (4.3),

$$
\rho_{\text {tach }}(v) \mathrm{d} v=\frac{8 \pi h}{c^{3}} \frac{\mathrm{d} v v^{2} \sqrt{v^{2}+m_{\mathrm{t}}^{2} c^{4} / h^{2}}}{\exp (\beta h v)-1} .
$$

A tachyon mass of $m_{\mathrm{t}} \approx 2.15 \mathrm{keV} / \mathrm{c}^{2}$ was found in Refs. [25,28], by comparing level shifts induced by the static potential of this field theory to high-precision Lamb shift measurements and QED calculations in hydrogen. The tachyon mass corresponds to a

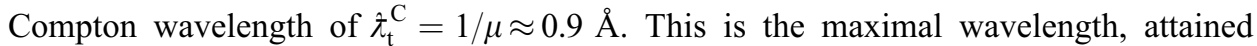
in the limit of infinite speed and zero energy, since $\lambda_{t}=\lambda_{t}{ }^{\mathrm{C}}\left(1+\left(\lambda_{t}{ }^{\mathrm{C}} v / c\right)^{2}\right)^{-1 / 2}$. As 
the tachyon radiation is in equilibrium with the photon background at $2.73 \mathrm{~K}$, we find $m_{\mathrm{t}} c^{2} /\left(k T_{0}\right) \approx 9.1 \times 10^{6}$ and $\beta h=1.76 \times 10^{-11} \mathrm{~s}$. The tachyon mass turns the frequency scaling in the Rayleigh-Jeans limit from quadratic to linear,

$$
\rho_{\text {tach }}(v \rightarrow 0) \sim \frac{8 \pi}{c h} m_{\mathrm{t}} v k T,
$$

Defining $x:=\beta h v$ and $\gamma:=m_{\mathrm{t}} c^{2} /(k T)$, we find the peak of $\rho_{\mathrm{tach}}(v)$ by solving

$$
\frac{x}{1-\mathrm{e}^{-x}}=2+\frac{x^{2}}{x^{2}+\gamma^{2}} \text {. }
$$

For the photon background, this means $x(\gamma=0) \approx 2.822$, and for the tachyon background $x(\gamma \rightarrow \infty) \approx 1.594$ applies, since, at the present epoch, $\gamma \approx 9.1 \times 10^{6}$, so that the tachyon mass does not really enter. Hence, the tachyonic energy density is peaked in the microwave range, at $v_{\mathrm{t}}^{\text {peak }} \approx 90.6 \mathrm{GHz}$, rather close to the peak of the photon density at $v_{\mathrm{ph}}^{\text {peak }} \approx 160 \mathrm{GHz}$.

The ratio of tachyon and photon transition rates is the same for spontaneous emission as well as for induced radiation, cf. (3.33), and (3.34). In dipole approximation,

$$
\frac{w^{\mathrm{d}(\mathrm{tach})}}{w^{\mathrm{d}(\mathrm{ph})}} \sim \frac{q^{2}}{e^{2}} \frac{\sqrt{(h v)^{2}+m_{\mathrm{t}}^{2} c^{4}}}{h v},
$$

so that $w^{\mathrm{d}(\text { tach })} / w^{\mathrm{d}(\mathrm{ph})} \approx 3.0 \times 10^{-9}$ for the Ly- $\alpha$ lines of hydrogen $(10.2 \mathrm{eV})$, based on $m_{\mathrm{t}} \approx 2.15 \mathrm{keV} / c^{2}$ and $q^{2} / e^{2} \approx 1.4 \times 10^{-11}$, cf. Refs. [25,28]. In heavy ions, it is even more unlikely that atomic transitions are effected by tachyon radiation; $w^{\mathrm{d}(\operatorname{tach})} / w^{\mathrm{d}(\mathrm{ph})} \approx$ $1.4 \times 10^{-11}$ for the Ly- $\alpha_{1}$ transition $(0.23 \mathrm{MeV})$ in hydrogenic uranium. In transitions between Rydberg states [40], the frequency dependent factor in (6.4) can get large, but then the dipole approximation is not valid any more. (In this case, the exponentials in (3.20) and (3.26) give rise to extensive averaging, because the tachyonic wavelength cannot supercede $\hat{\lambda}_{\mathrm{t}}^{\mathrm{C}}$, which is very small compared to Rydberg orbits with principal quantum number in the presently accessible range, $n \approx 10^{2-3}$.) Rydberg transitions lead to speedy tachyons, as the transition frequency relates to the velocity of the emitted tachyon via $h v=m_{\mathrm{t}} c^{2}\left(\mathbf{v}_{\mathrm{t}}^{2} / c^{2}-1\right)^{-1 / 2}$, and future high-precision measurements of Rydberg levels in hydrogenic systems could also result in a much more accurate determination of the tachyon mass and the tachyonic fine structure constant, since highly excited states are virtually unaffected by nuclear finite size effects, unlike ground state and $2 \mathrm{~S}-2 \mathrm{P}$ Lamb shifts and hyperfine transitions. The transition frequency of neighboring shells is $\Delta E_{n, n \pm 1} \sim 13.6 \mathrm{eV} \cdot 2 Z^{2} n^{-3}$, so that we find the tachyonic velocity as $\mathbf{v}_{\mathbf{t}} / c \sim 0.079 Z^{-2} n^{3}$, for low $Z$ and $n$ in the mentioned range and beyond.

The estimate (6.4) also applies to induced absorption rates for photonic and tachyonic cosmic background radiations, so that $w^{\mathrm{d}(\mathrm{tach})} / w^{\mathrm{d}(\mathrm{ph})}\left(v_{\mathrm{ph}}^{\text {peak }}\right) \approx 4.6 \times 10^{-5}$ at the maximum of the photon energy density at $2.73 \mathrm{~K}$. The chances to detect the cosmic tachyon radiation, i.e., to observe the limit (6.2) instead of the Rayleigh-Jeans law, improve with increasing wavelength, e.g., $w^{\mathrm{d}(\mathrm{tach})} / w^{\mathrm{d}(\mathrm{ph})}(7.3 \mathrm{MHz}) \approx 1$. The Planckian shape of the cosmic black-body radiation has not been tested below $0.5 \mathrm{GHz}$, cf. [41].

Next, we turn to the cross sections for the photoelectric effect and its tachyonic counterpart, cf. (3.43). As pointed out after (3.39), the validity of (3.43) is restricted 
to frequencies $E_{1} \ll h v \ll m_{\mathrm{e}} c^{2}$, due to the Born approximation and the non-relativistic treatment of the electron. For a hydrogenic ion, we have $E_{1} \approx 13.6 \mathrm{eV} \cdot Z^{2}$, and the mentioned restraints can be readily satisfied for light- and medium-sized ions, so that $k_{\mathrm{t}} / k_{\mathrm{e}} \ll 1$ is likewise satisfied, cf. (3.40). An upper bound for the ratio of tachyonic and photonic cross sections is evidently $\sigma^{\text {tach }} / \sigma^{\mathrm{ph}}<1.4 \times 10^{-11}$, attained for $h v \gg m_{\mathrm{t}} c^{2}$. In the opposite limit, this bound is reduced by a factor $h v / m_{\mathrm{t}} c^{2}$, which must be in turn much larger than $E_{1} / m_{\mathrm{t}} c^{2} \approx 6.5 \times 10^{-3} Z^{2}$. As for tachyonic Born scattering in hydrogenic Rydberg systems, studied at the end of Section 3, we have the mild restriction $v / c \gg \alpha / n, \alpha=Z / 137$, on the speed of the free electron. This implies $k_{\mathrm{t}} / k_{\mathrm{e}} \ll(n / \alpha) m_{\mathrm{t}} / m_{\mathrm{e}} \approx 0.6 n / Z$, which still admits $k_{\mathrm{t}} / k_{\mathrm{e}} \gg 1$ for large $n$. This limit is unattainable for photons in the non-relativistic Born approximation, where $k_{\mathrm{ph}} / k_{\mathrm{e}} \ll 1$ always applies.

Finally, we compare the ratio of photon and tachyon density as well as the ratio of the corresponding energy densities in the low-temperature regime, cf. (5.36). We find, with a background temperature of $2.73 \mathrm{~K}, N_{\text {tach }} / N_{\text {ph }} \approx 6.2 \times 10^{6}$ and $U_{\text {tach }} / U_{\text {ph }} \approx 3.4 \times 10^{6}$, but as we have demonstrated above, the high tachyon density cannot compensate the very small ratio $q^{2} / e^{2}$ of tachyonic and electric fine structure constants, at least not in the microwave regime. If we include longitudinal tachyons, these ratios are multiplied by a factor of $3 / 2$.

In this paper, we studied tachyonic emission and absorption processes semiclassically. The most promising approach to second quantization outside the light cone is still that of Feinberg [3], based on an incomplete set of eigenmodes (of a scalar field); the truncation of the momentum integration in the partition function (4.2) is in fact borrowed from Ref. [3], and it was semiclassically rederived in Section 3, using Einstein's argument of detailed balancing of emission and absorption rates. Feinberg's non-invariant vacuum is not an obstacle in an absolute cosmic space-time, if one defines it with respect to the comoving galaxy frame anchored in the cosmic ether [20,31-34], the local manifestation of the cosmic space-time. However, before one starts to endeavor on the quantization of the Proca field with negative mass square, one needs a good command of the classical theory, of wave propagation outside the light cone, and of interactions with subluminal currents. And above all, if one contemplates on tachyons, one has to decide where to search for them. In this paper and in Refs. [25,28] we scrutinized high-precision measurements in hydrogenic systems, Lamb shifts, hyperfine splittings, Rydberg transitions, tachyonic ionization cross sections, and the RayleighJeans limit of the cosmic tachyon background. Tachyonic cyclotron and synchrotron radiation, from storage rings to supernova remnants, will be discussed elsewhere.

\section{Acknowledgements}

The author acknowledges the support of the Japan Society for the Promotion of Science. I would like to thank C.V. Vishveshwara for initiating my visit to the Indian Institute of Astrophysics, Bangalore. The hospitality and stimulating atmosphere of 
the Centre for Non-linear Dynamics, Bharathidasan University, Trichy, the Institute of Mathematical Sciences, Madras, and the Tata Institute of Fundamental Research, Bombay, are likewise gratefully acknowledged. I'd like to thank Nandor Balazs and George Contopoulos for extensive discussions on causality.

\section{References}

[1] Th. Des Coudres, Arch. Néerland. Sci. (II) 5 (1900) 652.

[2] A. Sommerfeld, Proc. Konink. Akad. Wet. (Sec. Sci.) 7 (1904) 346.

[3] G. Feinberg, Phys. Rev. 159 (1967) 1089.

[4] G. Feinberg, Sci. Am. 222 (2) (1970) 69.

[5] G. Feinberg, Phys. Rev. D 17 (1978) 1651.

[6] P.A.M. Dirac, Proc. Roy. Soc. A 167 (1937) 148 (reprinted in: R.H. Dalitz (Ed.), The Collected Works of P.A.M. Dirac, 1924-1948, Cambridge UP, Cambridge, 1995).

[7] S. Tanaka, Prog. Theoret. Phys. 24 (1960) 171.

[8] Ya.P. Terletsky, Sov. Phys. Dokl. 5 (1961) 782.

[9] R. Newton, Science 167 (1970) 1569.

[10] P.C.W. Davies, Nuovo Cimento B 25 (1975) 571.

[11] E. Chaliasos, Physica A 144 (1987) 390.

[12] L. Brillouin, Wave propagation and group velocity, Academic Press, New York, 1960.

[13] J.A. Wheeler, R.P. Feynman, Rev. Mod. Phys. 17 (1945) 157.

[14] F. Hoyle, J.V. Narlikar, Rev. Mod. Phys. 67 (1995) 113.

[15] R.W. Fuller, J.A. Wheeler, Phys. Rev. 128 (1962) 919.

[16] D. Wycoff, N.L. Balazs, Physica A 146 (1987) 175.

[17] N.L. Balazs, A. Voros, Phys. Rep. 143 (1986) 109.

[18] R. Tomaschitz, Chaos Solitons Fractals 7 (1996) 753.

[19] R. Tomaschitz, Chaos Solitons Fractals 8 (1997) 761.

[20] R. Tomaschitz, Chaos Solitons Fractals 9 (1998) 1199.

[21] R. Tomaschitz, Int. J. Mod. Phys. D 7 (1998) 279.

[22] R. Anderson, I. Vetharaniam, G.E. Stedman, Phys. Rep. 295 (1998) 93.

[23] A. Proca, J. Phys. 7 (1936) 347.

[24] A.S. Goldhaber, M.M. Nieto, Rev. Mod. Phys. 43 (1971) 277.

[25] R. Tomaschitz, Int. J. Mod. Phys. A 14 (1999) 4275, 5137.

[26] R. Tomaschitz, Int. J. Mod. Phys. A 15 (2000) 3019.

[27] R. Tomaschitz, Class. Quant. Grav. 16 (1999) 3349.

[28] R. Tomaschitz, Eur. Phys. J. B 17 (2000) 523.

[29] N.N. Bogoliubov, D.V. Shirkov, Introduction to the Theory of Quantized Fields, Wiley, New York, 1980.

[30] E. Whittaker, A History of the Theories of Aether and Electricity, Thomas Nelson \& Sons, London, 1951.

[31] R. Tomaschitz, Int. J. Theoret. Phys. 37 (1998) 1121.

[32] R. Tomaschitz, Astrophys. Space Sci. 259 (1998) 255.

[33] R. Tomaschitz, Astrophys. Space Sci. 271 (2000) 181.

[34] R. Tomaschitz, Celest. Mech. Dyn. Astron. 77 (2000) 107.

[35] L.I. Schiff, Quantum Mechanics, McGraw-Hill, New York, 1968.

[36] S. Chandrasekhar, Radiative Transfer, Dover Publ., New York, 1960.

[37] R.C. Tolman, Relativity, Thermodynamics and Cosmology, Clarendon, Oxford, 1934.

[38] S. Chandrasekhar, An Introduction to the Study of Stellar Structure, Dover Publ., New York, 1967.

[39] E.T. Copson, Asymptotic Expansions, Cambridge University Press, Cambridge, 1965.

[40] T.F. Gallagher, Rydberg Atoms, Cambridge University Press, Cambridge, 1994.

[41] G.F. Smoot, D. Scott, D.E. Groom et al., Eur. Phys. J. C 15 (2000) 1. 